\title{
Ordered Expansions in Boson Amplitude Operators
}

\section{Citation}

Cahill, K. E., and R. J. Glauber. 1969. “Ordered Expansions in Boson Amplitude Operators.”

Physical Review 177 (5) (January 25): 1857-1881. doi:10.1103/physrev.177.1857.

\section{Published Version}

10.1103/physrev.177.1857

\section{Permanent link}

http://nrs.harvard.edu/urn-3:HUL.InstRepos:33087070

\section{Terms of Use}

This article was downloaded from Harvard University's DASH repository, and is made available under the terms and conditions applicable to Other Posted Material, as set forth at http:// nrs.harvard.edu/urn-3:HUL.InstRepos:dash.current.terms-of-use\#LAA

\section{Share Your Story}

The Harvard community has made this article openly available.

Please share how this access benefits you. Submit a story.

Accessibility 


\title{
Ordered Expansions in Boson Amplitude Operators*
}

\author{
K. E. Cahill $\dagger$ and R. J. Glauber \\ Lyman Laboratory of Physics, Harvard University, Cambridge, Massachusetts 02138
}

(Received 8 July 1968)

\begin{abstract}
The expansion of operators as ordered power series in the annihilation and creation operators $a$ and $a^{\dagger}$ is examined. It is found that normally ordered power series exist and converge quite generally, but that for the case of antinormal ordering the required $c$-number coefficients are infinite for important classes of operators. A parametric ordering convention is introduced according to which normal, symmetric, and antinormal ordering correspond to the values $s=+1,0,-1$, respectively, of an order parameter $s$. In terms of this convention it is shown that for bounded operators the coefficients are finite when $s>0$, and the series are convergent when $s>\frac{1}{2}$. For each value of the order parameter $s$, a correspondence between operators and $c$-number functions is defined. Each correspondence is one-to-one and has the property that the function $f(\alpha)$ associated with a given operator $F$ is the one which results when the operators $a$ and $a^{\dagger}$ occurring in the ordered power series for $F$ are replaced by their complex eigenvalues $\alpha$ and $\alpha^{*}$. The correspondence which is realized for symmetric ordering is the Weyl correspondence. The operators associated by each correspondence with the set of $\delta$ functions on the complex plane are discussed in detail. They are shown to furnish, for each ordering, an operator basis for an integral representation for arbitrary operators. The weight functions in these representations are simply the functions that correspond to the operators being expanded. The representation distinguished by antinormal ordering expresses operators as integrals of projection operators upon the coherent states, which is the form taken by the $P$ representation for the particular case of the density operator. The properties of the full set of representations are discussed and are shown to vary markedly with the order parameter $s$.
\end{abstract}

\section{INTRODUCTION}

A MBIGUITIES in the ordering of operator products were among the earliest questions to occur in the development of quantum mechanics. In more recent years we have come to understand a close relationship between particular types of measurements and the operator orderings best suited to describing them. In many quantum-mechanical problems, such as those of quantum optics, we are now concerned with the ordering of general operator functions rather than simple operator products.

In the present paper we shall discuss the representation of arbitrary operators that refer to a system which we choose, for simplicity, to have only a single degree of freedom. We shall describe this system in terms of a pair of complex operators, $a$ and $a^{\dagger}$, which we refer to as the annihilation and creation operators. These operators, which obey the commutation relation $\left[a, a^{\dagger}\right]=1$, play a fundamental role in descriptions of systems of harmonic oscillators and quantized fields. Operators with the same algebraic properties may be defined for a broad class of different dynamical systems by forming complex linear combinations of pairs of observables $q$ and $p$ that are canonically conjugate, $[q, p]=i \hbar$.

Because of ordering problems which arise from the noncommutativity of the operators $a$ and $a^{\dagger}$, the representation of operators is a considerably richer subject than the representation of $c$-number functions. We

\footnotetext{
* Supported in part by the National Bureau of Standards and by the Air Force Office of Scientific Research.

$\dagger$ Present address: National Research Council Postdoctoral Research Associate at the National Bureau of Standards, Washington, D. C.
}

examine several aspects of this structure in the present paper with particular emphasis upon the types of ordering that are most useful in the description of physical experiments. In the paper which follows, we shall apply this analysis to the closely related problem of expressing quantum-mechanical ensemble averages in forms that offer, as much as possible, the simplicity of classical ensemble averages. The present discussion of operator ordering will provide the basis for our discussion there of the $P$ representation, the Wigner distribution, and other ways of representing density operators.

It is conventional to distinguish the products $\left(a^{\dagger}\right)^{n} a^{m}$ and $a^{m}\left(a^{\dagger}\right)^{n}$ by calling the first normally ordered and the second antinormally ordered. In general an operator is said to be normally (antinormally) ordered if the operator $a$ stands always to the right (left) of the operator $a^{\dagger}$. It is clear that any polynomial in the operators $a$ and $a^{\dagger}$ may be cast into normally or antinormally ordered form by using the commutation relation a finite number of times.

A problem we discuss in detail is that of expanding an arbitrary operator as an ordered power series in the operators $a$ and $a^{\dagger}$. We show that virtually every operator of interest possesses a convergent power-series expansion in the normally ordered products $\left(a^{\dagger}\right)^{n} a^{m}$. Power-series expansions in the antinormally ordered products $a^{m}\left(a^{\dagger}\right)^{n}$ are, however, of considerably less generality. We show that for many well-behaved operators, ones that are bounded and of finite trace, the required $c$-number coefficients are infinite.

These two varieties of ordering have been discussed recently in a number of references and some discussion 
has also been given of other varieties of ordering. ${ }^{1-5}$ The emphasis of the work published to date has been principally upon the development of formal expressions for ordered operators rather than upon their explicit meaning. Since those meanings are not in all cases selfevident and we want in any case to understand their limitations, we have found it necessary to exercise some mathematical care in the development of our arguments.

In order to examine more closely the role of operator ordering in power-series expansions and to be able to interpolate between normal and antinormal ordering, we have been led to introduce a parametrized ordering convention. This convention associates with every complex number $s$ a unique way of ordering all products of the operators $a$ and $a^{\dagger}$. Normal ordering, antinormal ordering, and a type of ordering that is symmetric in the operators $a$ and $a^{\dagger}$ correspond to the values $s=+1$, -1 , and 0 , respectively, of the order parameter $s$. We are not attempting by introducing this continuum of orderings to deal with all possible forms of ordering nor do we know of any physical applications for arbitrarily ordered operator functions. We shall continue to center our attention on the three principal forms of ordering. By embedding them in a continuum of orderings, we provide for them a natural context for viewing their differences and interrelationships.

Our parametrized ordering convention enables us to consider the existence of ordered power-series expansions for intermediate orderings. We show that for bounded operators the required $c$-number series coefficients are finite whenever the ordering is closer to normal ordering then to antinormal ordering, i.e., for Res $>0$. We show as well that for such operators the power series converge, in a sense which we make precise in Sec. IV, when the ordering is closer to normal than to symmetric ordering, or, more precisely, for $\operatorname{Re} s>\frac{1}{2}+\frac{1}{2}(\operatorname{Im} s)^{2}$.

These ordered power-series expansions and the associated formulas for the $c$-number coefficients afford for every value of the order parameter $s$ a relatively direct type of correspondence between operators and $c$-number functions. The rule characterizing each correspondence is to replace the $s$-ordered products $\left\{\left(a^{\dagger}\right)^{n} a^{m}\right\}_{s}$ in the power-series expansion of a given operator by the monomials $\left(\alpha^{*}\right)^{n} \alpha^{m}$. The properties of these correspondences, which are one to one, depend markedly upon the order parameter $s$. The classes of operators for which they are appropriate and the types of functions which they associate with different classes of operators are discussed in Sec. VII. The correspond-

\footnotetext{
1 W. H. Louisell, Radiation and Noise in Quantum Electronics (McGraw-Hill Book Co., New York, 1965), pp. 104-119.

2 K. E. Cahill, thesis, Harvard University, 1967 (University Mricrofilms, Ann Arbor) (unpublished). Many of the results of the present paper are presented there.

${ }^{3}$ M. Lax and W. H. Louisell, J. Quantum Electron. QE3, 47 (1967).

${ }^{4}$ M. Lax, Phys. Rev. 172, 350 (1968).

${ }^{5}$ G. S. Agarwal and E. Wolf, Phys. Letters 26A, 485 (1968).
}

ence specified by $s=0$ and associated with symmetric ordering may be identified with a correspondence introduced by Weyl. ${ }^{6}$

Our main interest in these correspondences is the possibility of using the function associated with a given operator as a weight function in an integral representation of that operator. This possibility is realized in Secs. VI and VII, where for each correspondence we introduce a set of operators that forms a basis for such an integral representation. We discuss the properties of these representations and of their weight functions in Secs. VI-VIII.

We begin with two introductory sections. Section II summarizes the useful properties of the coherent states and of the displacement operators $\exp \left(\alpha a^{\dagger}-\alpha^{*} a\right)$. In Sec. III we discuss the properties of an integral representation for arbitrary operators that is based upon the displacement operators. This expansion, which is due to Weyl,${ }^{6}$ provides the basis for much of our subsequent analysis.

\section{COHERENT STATES AND DISPLACEMENT OPERATORS}

An important part of our discussion will be based upon the use of a particular set of quantum states. If the system being studied is the electromagnetic field, these are the states that describe completely coherent fields. The systematic use of such coherent states has been found particularly well suited to the solution of a number of quantum-electrodynamical problems and problems of other types involving harmonic-oscillator degrees of freedom. In this section we shall indicate how states of a corresponding type may be defined for a broad range of physical systems. This extension of the definition of coherent states furnishes the opportunity to review their properties briefly.

Let us consider for simplicity a dynamical system that is described by a single pair of Hermitian observables $q$ and $p$ which are canonically conjugate, $[q, p]$ $=i \hbar$, and have eigenvalues which range continuously from minus infinity to plus infinity. The operators we shall use in defining the coherent states are complex linear combinations of $q$ and $p$. Corresponding to any real parameter $\lambda$, different from zero, we may form the combinations

$$
\begin{aligned}
a & =(2 h)^{-1 / 2}\left(\lambda q+i \lambda^{-1} p\right), \\
a^{\dagger} & =(2 h)^{-1 / 2}\left(\lambda q-i \lambda^{-1} p\right) .
\end{aligned}
$$

These operators satisfy the familiar commutation relation

$$
\left[a, a^{\dagger}\right]=1
$$

and therefore possess the same algebraic properties as the operators associated with the complex amplitude of

\footnotetext{
${ }^{6} \mathrm{H}$. Weyl, The Theory of Groups and Quantum Mechanics (Dover Publications, Inc., New York, 1950), pp. 272-276.
} 
a harmonic oscillator or the photon annihilation and creation operators of quantum electrodynamics.

The operator $a$ possesses, as we shall see, a twodimensional continuum of eigenstates. Let us first note that it has a particularly simple eigenstate corresponding to the eigenvalue zero. If we denote this state by $|0\rangle$, then its defining equation

$$
a|0\rangle=0
$$

may be written in the representation in which $q$ is diagonal as

$$
\left(\lambda^{2} q^{\prime}+\hbar d / d q^{\prime}\right)\left\langle q^{\prime} \mid 0\right\rangle=0 .
$$

The wave function for this state is therefore a Gaussian function which takes the normalized form

$$
\left\langle q^{\prime} \mid 0\right\rangle=\left(\lambda^{2} / \pi \hbar\right)^{1 / 4} \exp \left[-\left(\lambda q^{\prime}\right)^{2} / 2 \hbar\right] .
$$

This state, incidentally, is one for which the uncertainty product $\Delta q \Delta p$ assumes its minimum value of $\frac{1}{2} h$ with the ratio $\Delta p / \Delta q=\lambda^{2}$.

It is clear from the algebraic properties of the operators $a$ and $a^{\dagger}$ that we may construct, just as in the case of the harmonic oscillator, a sequence of states for the system which correspond to integer eigenvalues for the product $a^{\dagger} a$. These states, which we label as $|n\rangle$, satisfy the equation

$$
a^{\dagger} a|n\rangle=n|n\rangle
$$

for $n=0,1,2, \cdots$. They may be generated from the state $|0\rangle$ by the rule

$$
|n\rangle=(n !)^{-1 / 2}\left(a^{\dagger}\right)^{n}|0\rangle .
$$

The wave functions $\left\langle q^{\prime} \mid n\right\rangle$ which represent these states are easily seen to take the same form as the familiar stationary-state wave functions for the harmonic oscillator. They are the Hermite functions which form a complete basis for the expansion of any quadratically integrable function. These states $|n\rangle$ therefore form a complete set.

It may be noted that no specification has been made of the Hamiltonian of the system that we are discussing. Since the system may have arbitrary dynamical behavior, the states $|n\rangle$ will not in general be energy eigenstates. Only when the Hamiltonian is a function of the operator

$$
a^{\dagger} a=(2 \hbar)^{-1}\left(\lambda^{-2} p^{2}+\lambda^{2} q^{2}-h\right)
$$

will the states $|n\rangle$ be stationary. The Hamiltonian of a harmonic oscillator of mass $m$ and angular frequency $\omega$ is

$$
H=(2 m)^{-1}\left(p^{2}+m^{2} \omega^{2} q^{2}\right) .
$$

The states $|n\rangle$ become the stationary states of this Hamiltonian when the arbitrary parameter $\lambda$ is given the value $(m \omega)^{1 / 2}$. A normal mode of the electromagnetic field in a dielectric possesses a Hamiltonian of the form of Eq. (2.9) with $\lambda=\sqrt{ }(\epsilon \omega)$, where $\epsilon$ is the dielectric constant. In this case the state $|n\rangle$ is an $n$-photon state.
Let us now define for each complex number $\alpha$ the exponential operator ${ }^{7}$

$$
D(\alpha)=\exp \left(\alpha a^{\dagger}-\alpha^{*} a\right),
$$

which is unitary and obeys the relation

$$
D^{\dagger}(\alpha)=D^{-1}(\alpha)=D(-\alpha) .
$$

These operators, which were introduced by Weyl ${ }^{6}$ are easily written in forms in which the operator $a$ appears exclusively to the right or to the left of the operator $a^{\dagger}$. By means of the identity ${ }^{8}$

$$
\exp A \exp B=\exp \left(A+B+\frac{1}{2}[A, B]\right),
$$

which holds whenever the commutator $[A, B]$ commutes with both $A$ and $B$, we find as the normally ordered form

$$
D(\alpha)=e^{-|\alpha|^{2} / 2} e^{\alpha{ }^{\dagger}} e^{-\alpha^{*} a}
$$

and as the antinormally ordered form

$$
D(\alpha)=e^{|\alpha|^{2} / 2} e^{-\alpha^{*} a} e^{\alpha a \dagger} .
$$

By differentiating these two ordered forms of the operators $D(\alpha)$ with respect to $-\alpha^{*}$, we find ${ }^{9}$

$$
\begin{aligned}
-\partial / \partial \alpha^{*} D(\alpha) & =D(\alpha)\left(a+\frac{1}{2} \alpha\right) \\
& =\left(a-\frac{1}{2} \alpha\right) D(\alpha),
\end{aligned}
$$

which implies the relation

and its adjoint

$$
D^{-1}(\alpha) a D(\alpha)=a+\alpha
$$

$$
D^{-1}(\alpha) a^{\dagger} D(\alpha)=a^{\dagger}+\alpha^{*} .
$$

Because of their property of displacing the operators $a$ and $a^{\dagger}$ in a conjugate fashion, the operators $D(\alpha)$ have been called displacement operators.

It is evident from their definition that the displacement operators obey a simple multiplication law. By applying the identity $(2.13)$ we find

$$
D(\alpha) D(\beta)=D(\alpha+\beta)^{\prime} \exp \left[\frac{1}{2}\left(\alpha \beta^{*}-\alpha^{*} \beta\right)\right] .
$$

Thus, apart from unimodular phase factors, the displacement operators form an Abelian group.

For each complex number $\alpha$ the coherent state $|\alpha\rangle$ is defined by ${ }^{7}$

$$
|\alpha\rangle=D(\alpha)|0\rangle .
$$

From Eqs. (2.4) and (2.17) it is clear that the state $|\alpha\rangle$ is an eigenstate of the operator $a$ with eigenvalue $\alpha$,

$$
a|\alpha\rangle=\alpha|\alpha\rangle
$$

The displacement transformation (2.20) leaves the variances of the coordinate and momentum variables unaltered so that they have for all coherent states the

\footnotetext{
7 R. J. Glauber, Phys. Rev. 131, 2766 (1963).

8 A. Messiah, Quantum Mechanics (North-Holland Publishing Co., Amsterdam, 1961), Vol. I, p. 442.

In this and in all subsequent differentiations with respect to conjugate complex variables we observe the convention $\partial \alpha / \partial \alpha^{*}$ $=\partial \alpha^{*} / \partial \alpha=0$.
} 
values

$$
(\Delta p)^{2}=\lambda^{4}(\Delta q)^{2}=\frac{1}{2} \lambda^{2} h
$$

which are characteristic of the ground state (2.6).

By using Eqs. (2.4), (2.8), (2.14), and (2.20), we may relate the coherent states to the states $|n\rangle$ :

$$
\begin{aligned}
|\alpha\rangle & =D(\alpha)|0\rangle \\
& =e^{-|\alpha|^{2} / 2} e^{\alpha a \dagger} e^{-\alpha^{*} a}|0\rangle \\
& =e^{-|\alpha|^{2} / 2} e^{\alpha a \dagger}|0\rangle \\
& =e^{-|\alpha|^{2} / 2} \sum_{n=0}^{\infty}(n !)^{-1 / 2} \alpha^{n}|n\rangle .
\end{aligned}
$$

This expansion and the orthonormality of the basis states $|n\rangle$ allow us to write the scalar product $\langle\beta \mid \alpha\rangle$ in the form

$$
\langle\beta \mid \alpha\rangle=\exp \left(-\frac{1}{2}|\alpha|^{2}-\frac{1}{2}|\beta|^{2}+\beta^{*} \alpha\right),
$$

which shows that no two coherent states are orthogonal. The extent to which they overlap

$$
|\langle\beta \mid \alpha\rangle|^{2}=e^{-|\alpha-\beta|^{2}}
$$

is, however, negligibly small when the states are macroscopically distinguishable, i.e., when $|\alpha-\beta| \gg 1$.

The coherent states provide a convenient representation for the unit or identity operator 1. Let us define as a real element of area in the complex $\alpha$ plane

$$
\pi^{-1} d^{2} \alpha=\pi^{-1} d(\operatorname{Re} \alpha) d(\operatorname{Im} \alpha),
$$

which, by writing

$$
d \alpha=(2 \hbar)^{-1 / 2}\left(\lambda d q^{\prime}+i \lambda^{-1} d p^{\prime}\right)
$$

we may recognize as the familiar differential element of phase space,

$$
\pi^{-1} d^{2} \alpha=h^{-1} d q^{\prime} d p^{\prime}
$$

Then on using the expansion (2.23) and integrating over the complex plane, we find

$$
\begin{aligned}
\int|\alpha\rangle\langle\alpha| \pi^{-1} d^{2} \alpha & =\sum_{n=0}^{\infty}|n\rangle\langle n| \\
& =1
\end{aligned}
$$

which illustrates the completeness of the coherent states. This relation affords for the trace of an arbitrary operator $F$ the simple expression

$$
\begin{aligned}
\operatorname{Tr}[F] & =\operatorname{Tr}\left[F \int|\alpha\rangle\langle\alpha| \pi^{-1} d^{2} \alpha\right] \\
& =\int\langle\alpha|F| \alpha\rangle \pi^{-1} d^{2} \alpha .
\end{aligned}
$$

\section{COMPLETENESS OF DISPLACEMENT OPERATORS}

The displacement operators $D(\alpha)$, which were defined in Sec. II, possess a number of simple properties which will be particularly useful in the analysis which follows. Principal among these is the representation that they afford for a certain class of operators as weighted integrals with square-integrable weight functions. In this representation, as we shall see, they play a role very much analogous to that of the unimodular exponential functions in the Fourier integral representation of square-integrable functions. Although the completeness property has only recently been formulated rigorously, ${ }^{2,10}$ the displacement operators have often been discussed in the literature. ${ }^{6,7,11-16}$ Because our analysis is cast in terms of the complex operators $a$ and $a^{\dagger}$, as opposed to their real parts $q$ and $p$, we shall need a notation for the Fourier transform that is more suited to complex numbers than to pairs of real numbers. If $g(\xi)$ is a function of the complex variable $\xi$, then we define its complex Fourier transform $f(\alpha)$ by the relation

$$
f(\alpha)=\int e^{\alpha \xi^{*}-\alpha^{*} \xi} g(\xi) \pi^{-1} d^{2} \xi
$$

where the integration is over the whole $\xi$ plane, and the element $d^{2} \xi$ is defined by Eq. (2.26). By letting $\alpha=x+i y$ and $\xi=u+i v$, we may write $f(\alpha)$ in the form

$$
f(x+i y)=\int_{-\infty}^{\infty} \int_{-\infty}^{\infty} e^{2 i(y u-x v)} g(u+i v) \pi^{-1} d u d v,
$$

from which it is evident that $f(\alpha)$ differs from the usual Fourier transform only by a scale change of its arguments $\operatorname{Re} \alpha$ and $\operatorname{Im} \alpha$.

By recalling the familiar formula for the Fourier integral representation of the product of two $\delta$-functions

$$
\delta(x) \delta(y)=\int_{-\infty}^{\infty} \int_{-\infty}^{\infty} e^{i\left(x k+y k^{\prime}\right)} \frac{d k d k^{\prime}}{(2 \pi)^{2}}
$$

we may express the two-dimensional $\delta$-function

$$
\delta^{(2)}(\alpha)=\delta(\operatorname{Re} \alpha) \delta(\operatorname{Im} \alpha)
$$

in the more convenient form

$$
\delta^{(2)}(\alpha)=\int e^{\alpha \xi^{*}-\alpha^{*} \xi} \pi^{-2} d^{2} \xi
$$

${ }^{10}$ J. C. T. Pool, J. Math. Phys. 7, 66 (1966).

11 J. E. Moyal, Proc. Cambridge Phil. Soc. 45, 99 (1948).

${ }_{12}$ M. S. Bartlett and J. E. Moyal, Proc. Cambridge Phil. Soc. 45, 545 (1949).

${ }_{13}$ U. Fano, Rev. Mod. Phys. 29, 74 (1957).

${ }^{14}$ J. Schwinger, Proc. Nat. Acad. Sci. (U.S.) 46, 883 (1960).

15 A. E. Glassgold and D. Holliday, Phys. Rev. 139, A1717 (1965).

${ }_{16} \mathrm{~J}$. R. Klauder, J. McKenna, and D. G. Currie, J. Math. Phys. 6, 743 (1965). 
In the present notation the processes of Fourier transformation and Fourier inversion are completely symmetrical. For, as is easily demonstrated with the aid of Eq. (3.3), if the functions $f(\alpha)$ and $g(\alpha)$ stand in the relationship

$$
f(\alpha)=\int e^{\alpha \xi^{*}-\alpha^{*} \xi} g(\xi) \pi^{-1} d^{2} \xi
$$

then the inverse relationship is

$$
g(\alpha)=\int e^{\alpha \xi^{*}-\alpha^{*} \xi} f(\xi) \pi^{-1} d^{2} \xi .
$$

Thus, if $f(\alpha)$ is the complex Fourier transform of $g(\alpha)$, then $g(\alpha)$ is the complex Fourier transform of $f(\alpha)$

The form which other familiar relations assume in this notation may be similarly derived through the use of the definition (3.1) and Eq. (3.3). If the functions $f_{1}(\alpha)$ and $f_{2}(\alpha)$ are the complex Fourier transforms of the functions $g_{1}(\xi)$ and $g_{2}(\xi)$, then we have

$$
\int f_{1}^{*}(\alpha) f_{2}(\alpha) d^{2} \alpha=\int g_{1}^{*}(\xi) g_{2}(\xi) d^{2} \xi
$$

and

$$
\int f_{1}(\alpha) f_{2}(\alpha) d^{2} \alpha=\int g_{1}(\xi) g_{2}(-\xi) d^{2} \xi
$$

Let us digress for a moment and remind ourselves of the class of functions to which the definition (3.1) and the relations (3.4)-(3.7) apply. This class, which is known as the Lebesgue class $L_{2}$, consists of all ordinary (i.e., measureable) functions $f(\alpha)$ for which the norm $\|f\|$ defined by

$$
\|f\|=\left(\int|f(\alpha)|^{2} \pi^{-1} d^{2} \alpha\right)^{1 / 2}
$$

is finite. ${ }^{17,18}$ (For our purposes the term measurable may be largely ignored, and the terms $L_{2}$ and square-integrable used interchangeably.) Under the operation of Fourier transformation the class of $L_{2}$ functions is mapped onto itself in a one-to-one fashion. If in Eq. (3.4) the function $g(\xi)$ is in $L_{2}$, so is $f(\alpha)$, and the inverse relation (3.5) is valid. If in Eqs. (3.6) and (3.7) the functions $g_{1}(\xi)$ and $g_{2}(\xi)$ are in $L_{2}$, then so are the functions $f_{1}(\alpha)$ and $f_{2}(\alpha)$ and Eqs. (3.6) and (3.7) hold. For $L_{2}$ functions the Schwarz inequality takes the form

$$
\left|\int f(\alpha) g(\alpha) \pi^{-1} d^{2} \alpha\right| \leq \int|f(\alpha) g(\alpha)| \pi^{-1} d^{2} \alpha
$$

$$
\leq\|f\|\|g\| \text {. }
$$

\footnotetext{
${ }^{17}$ W. Rudin, Principles of Mathematical Analysis (McGrawHill Book Co., New York, 1953). In Chap. 10 the Lebesgue theory is discussed in elementary terms.

${ }^{18} \mathrm{~S}$. Bochner and K. Chandrasekharan, Fourier Transforms (Princeton University Press, Princeton, New Jersey, 1949), Chap. 4.
}

The notation that we have introduced may be used to derive a useful property of the traces of the displacement operators. By using the trace relation (2.28) we may write the trace of the operator $D(\alpha)$ in the form

$$
\operatorname{Tr}[D(\alpha)]=\int\langle\xi|D(\alpha)| \xi\rangle \pi^{-1} d^{2} \xi
$$

If we now write the displacement operator in its normally ordered form (2.14) and employ the eigenvalue property of the coherent states, we find

$$
\begin{aligned}
\operatorname{Tr}[D(\alpha)] & =e^{-|\alpha|^{2} / 2} \int\left\langle\xi\left|e^{\alpha a^{\dagger}} e^{-\alpha^{*} a}\right| \xi\right\rangle \pi^{-1} d^{2} \xi \\
& =e^{-|\alpha|^{2} / 2} \int e^{\alpha \xi^{*}-\alpha^{*} \xi} \pi^{-1} d^{2} \xi
\end{aligned}
$$

By referring to the $\delta$-function formula (3.3), we recognize this as

$$
\operatorname{Tr}[D(\alpha)]=\pi e^{-|\alpha|^{2} / 2 \delta^{(2)}}(\alpha)
$$

or, since the exponential is unity at $\alpha=0$,

$$
\operatorname{Tr}[D(\alpha)]=\pi \delta^{(2)}(\alpha) .
$$

From this relation and the multiplication law (2.19), we find

$$
\operatorname{Tr}\left[D(\alpha) D^{-1}(\beta)\right]=\pi \delta^{(2)}(\alpha-\beta),
$$

which may be viewed as a species of orthogonality rule for the displacement operators. ${ }^{15}$

Before presenting a derivation of the completeness property of the displacement operators, we shall use their orthogonality to suggest the main features of their completeness. Let us suppose that the operator $F$ and the function $f(\xi)$ stand in the relation

$$
F=\int f(\xi) D^{-1}(\xi) \pi^{-1} d^{2} \xi
$$

Then we may write

$$
\operatorname{Tr}[F D(\alpha)]=\int f(\xi) \operatorname{Tr}\left[D^{-1}(\xi) D(\alpha)\right] \pi^{-1} d^{2} \pi
$$

which, by virtue of the orthogonality rule (3.10), implies that

$$
f(\alpha)=\operatorname{Tr}[F D(\alpha)] .
$$

To see whether this solution is well behaved, we write

$$
\begin{aligned}
\operatorname{Tr}\left[F^{\dagger} F\right] & =\int f(\xi) \operatorname{Tr}\left[F^{\dagger} D^{-1}(\xi)\right] \pi^{-1} d^{2} \xi \\
& =\int|f(\xi)|{ }^{2} \pi^{-1} d^{2} \xi
\end{aligned}
$$


which implies that the function $f(\xi)$ is square-integrable when the trace $\operatorname{Tr}\left[F^{\dagger} F\right]$ is finite. This trace is closely related to the Hilbert-Schmidt norm $\|F\|$ for the operator $F$ which is defined by

$$
\|F\|=\left[\operatorname{Tr}\left(F^{\dagger} F\right)\right]^{1 / 2} .
$$

We shall say that an operator is bounded if this norm is finite. With this terminology, we may draw from Eqs. (3.12)-(3.14) the conclusion that if a bounded operator $F$ possesses the expansion (3.12), then the weight function $f(\xi)$ is given by Eq. (3.13), is unique, is in $L_{2}$, and has the same norm as the operator $F$, i.e.,

$$
\|f\|=\|F\| \text {. }
$$

We shall now derive the expansion (3.12) and several other identities. Our method will utilize the properties of the matrix elements of the displacement operators between coherent states. Let us denote by the symbol $I(\alpha, \beta, \gamma, \delta)$ the integral

$$
I(\alpha, \beta, \gamma, \delta)=\int\langle\beta|D(\xi)| \alpha\rangle\langle\gamma|D(-\xi)| \delta\rangle \pi^{-1} d^{2} \xi
$$

We note that by writing the displacement operators in their normally ordered form (2.14) and using the eigenvalue property of the coherent states, we may write this integral in the form

$$
\begin{aligned}
I(\alpha, \beta, \gamma, \delta)= & \langle\beta \mid \alpha\rangle\langle\gamma \mid \delta\rangle \\
& \times \int \exp \left[\xi(\beta-\gamma)^{*}-\xi^{*}(\alpha-\delta)-|\xi|^{2}\right] \pi^{-1} d^{2} \xi
\end{aligned}
$$

The Gaussian integral which occurs here occurs in a number of other places in the course of this paper; it is evaluated explicitly in Appendix A, Eq. (A2). By applying that result we find

$$
I(\alpha, \beta, \gamma, \delta)=\langle\beta \mid \alpha\rangle\langle\gamma \mid \delta\rangle \exp \left[(\beta-\gamma)^{*}(\delta-\alpha)\right] .
$$

If we simplify this expression by using formula (2.24) for the scalar product of two coherent states, we find

$$
I(\alpha, \beta, \gamma, \delta)=\langle\gamma \mid \alpha\rangle\langle\beta \mid \delta\rangle
$$

and so obtain the integral identity

$$
\langle\gamma \mid \alpha\rangle\langle\beta \mid \delta\rangle=\int\langle\beta|D(\xi)| \alpha\rangle\langle\gamma|D(-\xi)| \delta\rangle \pi^{-1} d^{2} \xi
$$

which holds for all complex numbers $\alpha, \beta, \gamma$, and $\delta$.

Let us now recall that a matrix element identity of the form $\langle b|F| c\rangle=\langle b|G| c\rangle$, if it holds for a complete set of states $|b\rangle,|c\rangle, \cdots$, is equivalent to the corresponding operator equation $F=G$. Thus, since the coherent states form a complete set of states as shown by Eq. (2.27), we see that the integral identity (3.18) implies the operator identities

$$
|\alpha\rangle\langle\beta|=\int\langle\beta|D(\xi)| \alpha\rangle D(-\xi) \pi^{-1} d^{2} \xi
$$

and

$$
1\langle\gamma \mid \alpha\rangle=\int D(\xi)|\alpha\rangle\langle\gamma| D(-\xi) \pi^{-1} d^{2} \xi
$$

where 1 is the identity operator.

We now note that by using twice the resolution (2.27) of the identity operator we may express an arbitrary bounded operator $F$ as an integral over the coherentstate dyadics $|\alpha\rangle\langle\beta|$ in the form

$$
F=\int|\alpha\rangle\langle\beta|\langle\alpha|F| \beta\rangle \pi^{-2} d^{2} \alpha d^{2} \beta .
$$

Thus if we substitute in this integral the expression (3.19) for the dyadic $|\alpha\rangle\langle\beta|$, we arrive at the expansion

$$
F=\int\langle\alpha|F| \beta\rangle\langle\beta|D(\xi)| \alpha\rangle D(-\xi) \pi^{-3} d^{2} \xi d^{2} \alpha d^{2} \beta
$$

and by using Eqs. (2.27) and (2.28) we find

$$
\begin{aligned}
F & =\int\langle\alpha|F D(\xi)| \alpha\rangle D(-\xi) \pi^{-2} d^{2} \xi d^{2} \alpha \\
& =\int \operatorname{Tr}[F D(\xi)] D(-\xi) \pi^{-1} d^{2} \xi,
\end{aligned}
$$

which is the expansion (3.12). This argument concludes our derivation of the completeness property of the displacement operators.

Let us now observe that by multiplying both sides of expansion (3.22) by an arbitrary bounded operator $G$ and forming the trace, we obtain the identity

$$
\operatorname{Tr}[F G]=\int \operatorname{Tr}[F D(\xi)] \operatorname{Tr}[G D(-\xi)] \pi^{-1} d^{2} \xi .
$$

When the operators $F$ and $G$ are both bounded, the lefthand side of this equation is finite because of the Schwarz inequality

$$
|\operatorname{Tr}(F G)| \leq\|F\|\|G\|
$$

By referring to the inequality (3.9) and Eqs. (3.13) and (3.16), we may impose the same upper bound upon the right-hand side of Eq. (3.23).

If we multiply both sides of the identity (3.20) by the coherent-state matrix element $\langle\alpha|F| \gamma\rangle$ of an arbitrary operator $F$ and integrate over $\alpha$ and $\gamma$ by using Eqs. (2.27) and (2.28), then we arrive at the identity

$$
1 \operatorname{Tr}[F]=\int D(\xi) F D^{-1}(\xi) \pi^{-1} d^{2} \xi
$$


By multiplying both sides of this equation by an arbitrary operator $G$, we may obtain the relations

$$
G \operatorname{Tr}[F]=\int G D(\xi) F D^{-1}(\xi) \pi^{-1} d^{2} \xi
$$

and

$$
\operatorname{Tr}[G] \operatorname{Tr}[F]=\int \operatorname{Tr}\left[G D(\xi) F D^{-1}(\xi)\right] \pi^{-1} d^{2} \xi .
$$

Let us now observe that if some set of states $|a\rangle$, $|b\rangle,|c\rangle, \cdots$ forms a complete orthonormal set, then the matrix elements $\langle a|D(\xi)| b\rangle \cdots$ form a complete orthonormal set of functions. For if we let $F=|c\rangle\langle d|$ and $G=|b\rangle\langle a|$ then Eq. (3.27) becomes

$$
\begin{aligned}
\delta_{a, b} \delta_{c, d} & =\int\langle a|D(\xi)| c\rangle\left\langle d\left|D^{-1}(\xi)\right| b\right\rangle \pi^{-1} d^{2} \xi \\
& =\int\langle a|D(\xi)| c\rangle\langle b|D(\xi)| d\rangle^{*} \pi^{-1} d^{2} \xi,
\end{aligned}
$$

which verifies the orthonormality property. The statement of completeness follows from Eq. (3.11), which, when two factors of the identity operator in the form

$$
1=\sum_{a}|a\rangle\langle a|
$$

are inserted, becomes

$$
\sum_{a, b}\langle a|D(\xi)| b\rangle\left\langle a\left|D\left(\xi^{\prime}\right)\right| b\right\rangle^{*}=\pi \delta^{(2)}\left(\xi-\xi^{\prime}\right) .
$$

As we show in Appendix B, when the orthonormal states are the basis states $|n\rangle$, the matrix elements $\langle m|D(\xi)| n\rangle$ assume the form

$\langle m|D(\xi)| n\rangle=\left(\frac{n !}{m !}\right)^{1 / 2} \xi^{m-n} e^{-|\xi|^{2} / 2} L_{n}^{(m-n)}\left(|\xi|^{2}\right)$,

where $L_{\boldsymbol{q}}{ }^{(p)}(x)$ is an associated Laguerre polynomial. ${ }^{19}$

Let us now return to the expansion (3.12) which, as we have seen, affords a one-to-one correspondence

$$
F \leftrightarrow f(\xi)=\operatorname{Tr}[F D(\xi)]
$$

between the class of all bounded operators $F$ and the class $L_{2}$ of all square-integrable functions $f(\xi)$. In view of Eq. (3.16) this correspondence is norm-preserving in the sense that the $L_{2}$ norm $\|f\|$ of the weight function $f(\xi)$ is equal to the Hilbert-Schmidt norm $\|F\|$ of the operator $F$ being expanded.

If we write the expansion (3.12) in the form

$$
F=\int \exp \left(a \xi^{*}-a^{\dagger} \xi\right) \operatorname{Tr}[F D(\xi)] \pi^{-1} d^{2} \xi,
$$

${ }^{19} \mathrm{~W}$. Magnus and F. Oberhettinger, Formulas and Theorems for the Functions of Mathematical Physics (Chelsea Publishing Co., New York, 1954), p. 85. then we may draw several parallels between it and the complex Fourier transform expansion (3.1) of an $L_{2}$ function $f(\alpha)$. We may observe that the operators $a$ and $a^{\dagger}$ in Eq. (3.32) correspond to the variables $\alpha$ and $\alpha^{*}$ in Eq. (3.1) and that the unitary operator $\exp \left(a \xi^{*}-a^{\dagger} \xi\right)$ in Eq. (3.32) plays the role of the unimodular function $\exp \left(\alpha \xi^{*}-\alpha^{*} \xi\right)$ in Eq. (3.1). The weight function $\operatorname{Tr}[F D(\xi)]$ corresponds to the function $g(\xi)$ and may therefore be thought of as a species of Fourier transform for the operator $F$. Just as the ordinary Fourier-transform expansion (3.1) induces a one-to-one correspondence $f(\alpha) \leftrightarrow g(\xi)$ from $L_{2}$ onto itself that is norm-preserving, $\|f\|=\|g\|$, so too the correspondence (3.31), between $L_{2}$ and the class of all bounded operators is one-to-one and norm-preserving.

So far we have been talking about the expansion of operators that are bounded. In the remainder of this section we shall say a few things about the expansion of other types of operators.

We have been using, and shall continue to use, the term bounded to denote operators for which the HilbertSchmidt norm $\|F\|=\left[\operatorname{Tr}\left(F^{\dagger} F\right)\right]^{1 / 2}$ is finite. Another operator norm which is frequently used and which we shall denote by $\|F\|_{1}$ is the least upper bound (l.u.b.), taken over all states $|\psi\rangle$ of unit norm $\langle\psi \mid \psi\rangle=1$, of the quantity $\left\langle\psi\left|F^{\dagger} F\right| \psi\right\rangle$, i.e.,

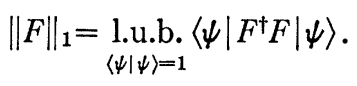

It is, loosely speaking, the largest eigenvalue of the operator $F^{\dagger} F$. Operators for which the norm $\|F\|_{1}$ is finite are often called bounded, but we shall call them finite. All bounded operators are finite, and we have $\|F\|_{1}$ $\leq\|F\|$. The most important unbounded but finite operators are the unitary operators for which $\|U\|_{1}=1$.

The displacement operator expansion (3.12) is not in general suited to the expansion of unbounded operators, even those that are finite. When the operator $F$ is unbounded, the appropriate weight function $\operatorname{Tr}[F D(\xi)]$, which must according to Eq. (3.14) lack square-integrability, is often singular. If, for example, we take as the operator $F$ the displacement operator $D(\alpha)$, which being unitary is finite, then the weight function $f(\xi)$ is given by Eq. (3.11) as $\pi \delta^{(2)}(\alpha+\xi)$. For this reason we shall not in this paper attempt a careful formulation of the representation of unbounded operators.

We may observe, however, that the coherent-state expansion (3.21) affords a representation for a broad class of unbounded operators. In this representation, as in the one afforded by the displacement operators, we find quadratic integrability linked with boundedness. For by using Eq. (3.21) to form the Hilbert-Schmidt norm of the operator $F$, we secure the result

$$
\|F\|^{2}=\operatorname{Tr}\left[F^{\dagger} F\right]=\int|\langle\alpha|F| \beta\rangle|^{2} \pi^{-2} d^{2} \alpha d^{2} \beta .
$$


The weight function $\langle\alpha|F| \beta\rangle$ of the representation (3.21) has no singularities in the finite $\alpha, \beta$ planes unless the operator $F$ is particularly pathological. ${ }^{7}$ Moreover, when $F$ is a finite operator the modulus of $\langle\alpha|F| \beta\rangle$ is bounded by $\|F\|_{1}$,

$$
|\langle\alpha|F| \beta\rangle| \leq\|F\|_{1},
$$

as may be seen from the definition (3.33).

For an arbitrary bounded operator $F$ the weight function $\operatorname{Tr}[F D(\xi)]$ need not be bounded or continuous. There is, however, a smaller class of operators for which both of these conditions are met. This is the trace class, also called the class of nuclear operators. The derivations of the statements that we shall now make about trace-class operators may be found in Refs. 2 and 20.

Every trace-class operator $F$ may be factored uniquely into the product of unitary operator $U$ and an operator $B$ which is positive-definite and of finite trace,

$$
F=U B \text {. }
$$

In terms of this decomposition, the trace-class norm is defined as

$$
\|F\|_{2}=\operatorname{Tr} B .
$$

Every density operator $\rho$ is a member of the trace class with $\|\rho\|_{2}=1$.

Every trace-class operator is bounded, as is shown by the inequality ${ }^{20}$

$$
\|F\|_{1} \leq\|F\| \leq\|F\|_{2}
$$

for the three norms which we have mentioned. The product of a finite operator $G$ and a trace-class operator $F$ is a trace-class operator and we have the inequality ${ }^{20}$

$$
|\operatorname{Tr} F G| \leq\|F\|_{2}\|G\|_{1} .
$$

From this relation it follows, since the displacement operators are finite with $\|D(\xi)\|_{1}=1$, that for every trace-class operator $F$ the weight function $\operatorname{Tr}[F D(\xi)]$ is uniformly bounded by the trace-class norm $\|F\|_{2}$

$$
|\operatorname{Tr}[F D(\xi)]| \leq\|F\|_{2} .
$$

It may also be shown ${ }^{2}$ that for every trace-class operator $F$ the weight function $\operatorname{Tr}[F D(\xi)]$ is a (uniformly) continuous function of $\xi$.

\section{ORDERED POWER-SERIES EXPANSIONS}

The completeness property of the displacement operators, which we discussed in Sec. III, affords a convenient framework for examining various ways in which bounded operators may be represented. In this section and in Sec. V, we discuss the problem of expanding a bounded operator as an ordered power series in the

${ }^{20}$ I. M. Gel'Fand and N. Ya. Vilenkin, Generalized Functions, translated by A. Feinstein (Academic Press Inc., New York, 1964), Vol. 4. Chap. 1, Sec. 2, is a detailed and readable account of the properties of trace-class operators and of Hilbert-Schmidt operators. operators $a$ and $a^{\dagger}$. The cases of normal ordering, of antinormal ordering, and of a type of ordering that is symmetric in the creation and annihilation operators occupy most of the present section. For these orderings we obtain closed integral expressions for the coefficients of the power-series expansions of an arbitrary bounded operator. It will become evident that there is a marked contrast between the properties of normally and antinormally ordered power-series expansions. We show that normally ordered power-series expansions exist and converge in a well-defined sense for a very broad class of operators. Antinormally ordered power series, however, do not afford a completely satisfactory representation for all bounded operators; we show that the appropriate coefficients are singular for large classes of bounded operators. We also consider briefly the expansion of bounded operators as ordered power series in the operators $q$ and $p$. Because these operators are Hermitian, the series coefficients, as we shall see, tend not to develop singularites.

To begin with, let us consider the possibility of expanding an arbitrary bounded operator $F$ as a normally ordered power series, i.e., in the form

$$
F=\sum_{n, m=0}^{\infty} c_{n, m}\left(a^{\dagger}\right)^{n} a^{m}
$$

where the coefficients $c_{n, m}$ are complex numbers. Since the operator $F$ is assumed to be bounded, it possesses the expansion (3.12),

$$
F=\int \operatorname{Tr}[F D(\xi)] D^{-1}(\xi) \pi^{-1} d^{2} \xi,
$$

where the weight function $\operatorname{Tr}[F D(\xi)]$ is square-integrable. We may formally generate the expansion (4.1) by writing the operator $D^{-1}(\xi)$ in its normally ordered form (2.14),

$$
F=\int \operatorname{Tr}[F D(\xi)] e^{-|\xi| 2 / 2} e^{-\xi a \dagger} e^{\xi * a} \pi^{-1} d^{2} \xi,
$$

and then expanding the exponentials in powers of $a$ and $a^{\dagger}$ so that we have

$$
\begin{aligned}
F=\sum_{n, m=0}^{\infty}\left(a^{\dagger}\right)^{n} a^{m} \frac{1}{n ! m !} \int \operatorname{Tr} & {[F D(\xi)] e^{-|\xi|^{2} / 2} } \\
& \times(-\xi)^{n}\left(\xi^{*}\right)^{m} \pi^{-1} d^{2} \xi .
\end{aligned}
$$

We may therefore identify the coefficients $c_{n, m}$ with the integrals

$$
\begin{aligned}
c_{n, m}=(n ! m !)^{-1} \int \operatorname{Tr}[F D(\xi)] e^{-|\xi|^{2 / 2}} & (-\xi)^{n} \\
& \times\left(\xi^{*}\right)^{m} \pi^{-1} d^{2} \xi .
\end{aligned}
$$

By using the inequality (3.9) and the relation (3.16), we find that for every bounded operator $F$ the coeffici- 
ents $c_{n, m}$ are finite and are bounded by the quantities

$$
\left|c_{n, m}\right| \leq(n ! m !)^{-1}[(n+m) !]^{1 / 2}\|F\|,
$$

where $\|F\|$ is the Hilbert-Schmidt norm (3.15).

The presence of the factor $e^{-|\xi|^{2} / 2}$ in the integral (4.5) for the coefficients $c_{n, m}$ suggests that normally ordered expansions may be appropriate for a larger class of operators than the one being considered. We shall take up this matter, as well as the question of the convergence of the series (4.1), after we have considered the cases of antinormal and symmetric order.

The coefficients $d_{n, m}$ of the antinormally ordered expansion

$$
F=\sum_{n, m=0}^{\infty} d_{n, m} a^{m}\left(a^{\dagger}\right)^{n}
$$

of an arbitrary bounded operator $F$ may be identified by an argument entirely similar to that of Eqs. (4.1)(4.5). If we write the operator $D^{-1}(\xi)$ in Eq. (4.1) in its antinormally ordered form (2.15), we have

$$
F=\int \operatorname{Tr}[F D(\xi)] e^{|\xi|^{2} / 2} e^{\xi^{*} a} e^{-\xi a \dagger} \pi^{-1} d^{2} \xi,
$$

and, by expanding the exponentials in powers of $a$ and $a^{\dagger}$, we find

$$
\begin{aligned}
d_{n, m}=(n ! m !)^{-1} \int \operatorname{Tr}[F D(\xi)] e^{|\xi|^{2} / 2} & (-\xi)^{n} \\
& \times\left(\xi^{*}\right)^{m} \pi^{-1} d^{2} \xi .
\end{aligned}
$$

This expression differs from the corresponding one (4.5) for the coefficients $c_{n, m}$ of the normally ordered series in that the exponential $e^{-|\xi|^{2 / 2}}$ has been replaced by $e^{+|\xi|^{2} / 2}$. Since for the class of bounded operators $F$ the weight functions $\operatorname{Tr}[F D(\xi)]$ form exactly the class $L_{2}$ of square-integrable functions, it is evident that the coefficients $d_{n, m}$ can become singular for certain classes of bounded operators. Examples of such operators are discussed at the end of this section.

So far we have made use of the normally and antinormally ordered expansions

and

$$
D(\alpha)=e^{-|\alpha|^{2} / 2} \sum_{n, m=0}^{\infty} \frac{\left(\alpha a^{\dagger}\right)^{n}}{n !} \frac{\left(-\alpha^{*} a\right)^{m}}{m !}
$$

$$
D(\alpha)=e^{|\alpha|^{2 / 2}} \sum_{n, m=0}^{\infty} \frac{\left(-\alpha^{*} a\right)^{m}}{m !} \frac{\left(\alpha a^{\dagger}\right)^{n}}{n !} .
$$

Let us now examine the expansion

$$
\begin{aligned}
D(\alpha) & =\exp \left(\alpha a^{\dagger}-\alpha^{*} a\right) \\
& =\sum_{n=0}^{\infty}(n !)^{-1}\left(\alpha a^{\dagger}-\alpha^{*} a\right)^{n},
\end{aligned}
$$

in which the operators $a$ and $a^{\dagger}$ are on an equal footing with respect to order. There are $(n+m) ! / n ! m !$ different ways of ordering the product of $n$ factors of $a^{\dagger}$ and $m$ factors of $a$. Let us denote by the symbol $\left\{a^{\dagger n} a^{m}\right\}$ the average of these $(n+m) ! / n ! m !$ differently ordered operator products.

Two examples of this average product, which we shall refer to as the symmetrically ordered product, ${ }^{2,21,22}$ are

and

$$
\left\{a^{\dagger} a\right\}=\frac{1}{2}\left(a^{\dagger} a+a a^{\dagger}\right)
$$

$$
\left\{a^{\dagger} a^{2}\right\}=\frac{1}{3}\left(a^{\dagger} a^{2}+a a^{\dagger} a+a^{2} a^{\dagger}\right) .
$$

In this notation we have

$$
\begin{aligned}
&\left(\alpha a^{\dagger}-\alpha^{*} a\right)^{n}=\sum_{r=0}^{n} \frac{n !}{r !(n-r) !} \alpha^{r}\left(-\alpha^{*}\right)^{n-r} \\
& \times\left\{\left(a^{\dagger}\right)^{r} a^{n-r}\right\},
\end{aligned}
$$

so that we may write the expansion (4.12) for the displacement operator as

$$
D(\alpha)=\sum_{n, m=0}^{\infty} \frac{\alpha^{n}\left(-\alpha^{*}\right)^{m}}{n ! m !}\left\{\left(a^{\dagger}\right)^{n} a^{m}\right\}
$$

By differentiating these relations we find for the operator $\left\{\left(a^{\dagger}\right)^{n} a^{m}\right\}$ the expression

$$
\left\{\left(a^{\dagger}\right)^{n} a^{m}\right\}=\left.\frac{\partial^{n+m} D(\alpha)}{\partial \alpha^{n} \partial\left(-\alpha^{*}\right)^{m}}\right|_{\alpha=0},
$$

which may be simplified to the form

$$
\left\{\left(a^{\dagger}\right)^{n} a^{m}\right\}=\left.\frac{\partial^{n+m}}{\partial x^{n} \partial y^{m}} \frac{\left(x a^{\dagger}+y a\right)^{n+m}}{(n+m) !}\right|_{x=y=0},
$$

where the variables $x$ and $y$ are set equal to zero after the differentiation.

We may now proceed as in the cases of normal and antinormal order to construct for an arbitrary bounded operator $F$ its symmetrically ordered power-series expansion,

$$
F=\sum_{n, m=0}^{\infty} b_{n, m}\left\{\left(a^{\dagger}\right)^{n} a^{m}\right\}
$$

When the expansion (4.14) is inserted into the general representation (4.2), we find

$$
\begin{aligned}
F=\sum_{n, m=0}^{\infty}\left\{\left(a^{\dagger}\right)^{n} a^{m}\right\}(n ! m !)^{-1} \int & \operatorname{Tr}[F D(\xi)] \\
& \times(-\xi)^{n}\left(\xi^{*}\right)^{m} \pi^{-1} d^{2} \xi,
\end{aligned}
$$

which implies that the coefficients $b_{n, m}$ are given by the

21 B. R. Mollow, Phys. Rev. 162, 1256 (1967).

${ }^{22} \mathrm{R}$. J. Glauber, in Proceedings of the Second International Summer School on Fundamental Problems of Statistical Mechanics, Noordwijk-aan-Zee, The Netherlands, 1967, edited by E. G. D. Cohen (North-Holland Publishing Co., Amsterdam, 1968). 
integrals

$b_{n, m}=(n ! m !)^{-1} \int \operatorname{Tr}[F D(\xi)](-\xi)^{n}\left(\xi^{*}\right)^{m} \pi^{-1} d^{2} \xi$.

The class of bounded operators $F$ for which these integrals converge consists therefore of those whose weight functions $\operatorname{Tr}[F D(\xi)]$ possess finite moments, While this is not the entire class of bounded operators, it is a much broader class than the class of operators for which the coefficients $d_{n, m}$ of the antinormally ordered expansions are finite. Speaking loosely, we might say that the coefficients $b_{n, m}$ tend to be finite for bounded operators.

Let us now return to the case of normal ordering and take up the question of convergence. A sequence of operators $A_{n}$ is said to converge weakly to the operator $A$ if for every pair of normalized states $|f\rangle$ and $|g\rangle$ the sequence $\left\langle f\left|A-A_{n}\right| g\right\rangle$ converges to zero. This type of convergence is clearly inappropriate as a definition for the convergence of power-series expansions. The state $|g\rangle$,

$$
|g\rangle=\frac{\sqrt{ } 6}{\pi} \sum_{n=0}^{\infty} \frac{1}{n}|n\rangle,
$$

for example, is normalized; but the quantities $\left\langle g\left|\left(a^{\dagger}\right)^{n} a^{m}\right| g\right\rangle$ are all infinite, except when $n+m<2$. Thus, the power-series expansions that we have discussed do not converge weakly unless they terminate.

We shall adopt for simplicity the following criterion ${ }^{2}$ for the convergence of ordered power-series expansions. The power series

$$
F=\sum_{n, m=0}^{\infty} f_{n, m}\left\{\left(a^{\dagger}\right)^{n} a^{m}\right\}_{\text {ord }},
$$

where the symbol $\left\{\left(a^{\dagger}\right)^{n} a^{m}\right\}_{\text {ord }}$ denotes an arbitrarily ordered product, will be said to converge if for every pair of coherent states $|\alpha\rangle$ and $|\beta\rangle$ we have

$$
\langle\beta|F| \alpha\rangle=\lim _{N, M \rightarrow \infty} \sum_{n=0}^{N} \sum_{m=0}^{M}\left\langle\beta\left|f_{n, m}\left\{\left(a^{\dagger}\right)^{n} a^{m}\right\}_{\text {ord }}\right| \alpha\right\rangle .
$$

In effect this is weak convergence over the set of coherent states. Since the coherent states form a complete set, an operator is defined uniquely by its power-series expansion if that expansion converges.

In terms of this criterion the question of the convergence of the normally ordered power series (4.1) is a simple one. Our task is to show that the sequence (4.21) converges to the matrix element $\langle\beta|F| \alpha\rangle$. The series in question is

$$
\langle\beta|F| \alpha\rangle=\langle\beta \mid \alpha\rangle \sum_{n, m=0}^{\infty} c_{n, m}\left(\beta^{*}\right)^{n} \alpha^{m} .
$$

We note that this series converges if and only if the function $\langle\beta|F| \alpha\rangle /\langle\beta \mid \alpha\rangle$ is an entire function of the variables $\beta^{*}$ and $\alpha$.
In order to examine the analyticity of this function, let us use Eq. (2.23) to write it in the form

$$
\frac{\langle\beta|F| \alpha\rangle}{\langle\beta \mid \alpha\rangle}=e^{-\beta^{*} \alpha} \sum_{n, m=0}^{\infty} \frac{\langle n|F| m\rangle}{(n ! m !)^{1 / 2}}\left(\beta^{*}\right)^{n} \alpha^{m} .
$$

This series converges and defines an entire function of $\beta^{*}$ and $\alpha$ for an extremely broad class of operators. This is the case, for example, when for some $M, R_{1}, R_{2}$, and $\epsilon>0$ the inequalities

$$
|\langle n|F| m\rangle| \leq M R_{1}{ }^{n} R_{2}{ }^{m}(n ! m !)^{1 / 2-\epsilon}
$$

are satisfied. Finite operators fulfill these conditions with $R_{1}=R_{2}=1, \epsilon=\frac{1}{2}$, and $M=\|F\|_{1}$.

We have shown that an operator $F$ possesses a convergent normally ordered power-series expansion when the series (4.22) converges and that the latter series converges when the operator $F$ satisfies the condition (4.23). The very general condition (4.23) is therefore a sufficient one for the convergence of the normally ordered power series (4.1).

It is easy to adapt the methods of this section to the expansion of bounded operators as power series in the Hermitian operators $q$ and $p$. To begin with, we must write the displacement operator $D(\alpha)$ in forms in which the operators $q$ and $p$ appear in definite orders. By using Eqs. (2.1), (2.2) and (2.13), and writing $\alpha=(2 \hbar)^{-1}$ $\times\left(\lambda q^{\prime}+i \lambda^{-1} p^{\prime}\right)$, we find, after putting $\hbar=1$,

$$
\begin{aligned}
D\left(q^{\prime}, p^{\prime}\right) \equiv D(\alpha) & =e^{i\left(q p^{\prime}-p q^{\prime}\right)} \\
& =e^{i q p^{\prime}} e^{-i p q^{\prime}} e^{-i q^{\prime} p^{\prime} / 2} \\
& =e^{-i p q^{\prime}} e^{i q p^{\prime}} e^{i q^{\prime} p^{\prime} / 2} .
\end{aligned}
$$

If we now write Eq. (4.2) if the form

$$
F=\int \operatorname{Tr}\left[F D\left(q^{\prime}, p^{\prime}\right)\right] e^{-i\left(q p^{\prime}-p q^{\prime}\right)}(2 \pi)^{-1} d q^{\prime} d p^{\prime},
$$

then we may derive in analogy with Eqs. (4.4), (4.9) and (4.18) three expansions for an arbitrary bounded operator $F$. These are what we may call the $p$-ordered power-series expansion

$$
\begin{array}{r}
F=\sum_{n, m=0}^{\infty} q^{n} p^{m}(n ! m !)^{-1} \int \operatorname{Tr}\left[F D\left(q^{\prime}, p^{\prime}\right)\right]\left(-i p^{\prime}\right)^{n} \\
\times\left(i q^{\prime}\right)^{m} e^{-i q^{\prime} p^{\prime}(2 \pi)^{-1} d q^{\prime} d p^{\prime},}
\end{array}
$$

the $q$-ordered expansion

$$
\begin{array}{r}
F=\sum_{n, m=0}^{\infty} p^{m} q^{n}(n ! m !)^{-1} \int \operatorname{Tr}\left[F D\left(q^{\prime}, p^{\prime}\right)\right]\left(-i p^{\prime}\right)^{n} \\
\times\left(i q^{\prime}\right)^{m} e^{i q^{\prime} p^{\prime}(2 \pi)^{-1} d q^{\prime} d p^{\prime},}
\end{array}
$$


and the $q$ - $p$-symmetric expansion

$$
\begin{aligned}
& F=\sum_{n, m=0}^{\infty}\left\{q^{n} p^{m}\right\}(n ! m !)^{-1} \int \operatorname{Tr}\left[F D\left(q^{\prime} p^{\prime}\right)\right]\left(-i p^{\prime}\right)^{n} \\
& \times\left(i q^{\prime}\right)^{m}(2 \pi)^{-1} d q^{\prime} d p^{\prime}
\end{aligned}
$$

where the $q$ - $p$-symmetric product is defined by analogy with Eq. (4.15) as

$$
\left\{q^{n} p^{m}\right\}=\left.i^{m-n} \frac{\partial^{n+m} D\left(q^{\prime}, p^{\prime}\right)}{\partial\left(p^{\prime}\right)^{n} \partial\left(q^{\prime}\right)^{m}}\right|_{q^{\prime}=p^{\prime}=0} .
$$

Because the commutator $[q, p]=i$ is purely imaginary, the integrals defining the coefficients of these expansions differ from one another only by unimodular factors in their integrands. A bounded operator that possesses one of the expansions (4.26)-(4.28) is therefore very likely to possess the other two. This class of operators is approximately the same as the class for which the symmetrically ordered expansion (4.17) is appropriate.

Let us now illustrate some of the results of this section by considering some simple examples. Our first one will serve to answer a question which was not raised in the foregoing discussion, namely, whether antinormally ordered expansions are suitable for trace-class operators (defined in Sec. III). The operator $|\alpha\rangle\langle\beta|$, which is the outer product of two coherent states, is both bounded and in the trace class. By using Eqs. (2.14) and (2.21), we find as its weight function the exponential

$\operatorname{Tr}[D(\xi)|\alpha\rangle\langle\beta|]=\langle\beta \mid \alpha\rangle \exp \left(-\frac{1}{2}|\xi|^{2}+\xi \beta^{*}-\xi^{*} \alpha\right)$.

Reference to Eqs. (4.5), (4.9) and (4.19) reveals that the coefficients of the normally and symmetrically ordered expansions are finite, while those of the antinormally ordered expansion are singluar.

Another operator which is in the trace class is the outer product $|n\rangle\langle m|$ of two states with fixed numbers of quanta. The appropriate weight function is

$$
\operatorname{Tr}[|n\rangle\langle m| D(\xi)]=\langle m|D(\xi)| n\rangle,
$$

and from the explicit formula (3.30) for this function and Eqs. (4.5), (4.9) and (4.19) it is evident that the coefficients $c_{n, m}$ and $b_{n, m}$ are finite, while the $d_{n, m}$ are singular. We conclude from these two examples that trace-class operators do not necessarily possess antinormally ordered power-series expansions.

As a more useful example let us consider the operator

$$
F(\lambda)=\lambda^{a^{\dagger a}}=\exp \left(a^{\dagger} a \ln \lambda\right)
$$

which depends analtyically upon the complex parameter $\lambda$ since the operator $a^{\dagger} a$ has integer eigenvalues. This operator is finite for $|\lambda| \leq 1$ and is both bounded and in the trace class for $|\lambda|<1$. The operator $F(\lambda)$ has many applications in thermal equilibrium statistics where we usually have $\lambda=\exp (-\hbar \omega / k T)<1$, where $k$ is the Boltzmann constant and $T$ is the temperature.
We may find directly the normally ordered power series for $F(\lambda)$ by differentiating it with respect to $\lambda$ :

$$
\frac{d F(\lambda)}{d \lambda}=a^{\dagger} a \lambda^{a \dagger a-1}
$$

Then since for any function $f$ we have

$$
a f\left(a^{\dagger} a\right)=f\left(a^{\dagger} a+1\right) a,
$$

we may write

$$
\begin{aligned}
d F(\lambda) / d \lambda & =a^{\dagger} \lambda^{a^{\dagger a} a} a \\
& =a^{\dagger} F(\lambda) a .
\end{aligned}
$$

The operator $F(\lambda)$ thus satisfies a differential equation whose solution is a normally ordered exponential function of $a^{\dagger} a$. The initial condition $F(1)=1$ is satisfied by the solution

$$
F(\lambda)=: e^{(\lambda-1) a \dagger a}:,
$$

where the symbol : : means that the exponential is in normally ordered form.

The coefficients $c_{n, m}$ of this expansion are given by

$$
c_{n, m}=\delta_{n, m}(n !)^{-1}(\lambda-1)^{n} \text {. }
$$

They are entire functions of $\lambda$. Since the operator $F(\lambda)$ as is easily shown, satisfies the conditions (4.23), its normally ordered expansion (4.35) converges for all $\lambda$.

By using Eq. (2.15), (2.28) and (4.35), we may write the weight function for $F(\lambda)$ in the form

$$
\begin{aligned}
\operatorname{Tr}[F(\lambda) D(\xi)] & =e^{|\xi|^{2} / 2} \operatorname{Tr}\left[e^{\xi a \dagger} F(\lambda) e^{-\xi^{*} a}\right] \\
= & e^{|\xi|^{2} / 2} \int \exp \left[\xi \alpha^{*}-\xi^{*} \alpha+(\lambda-1)|\alpha|^{2}\right] \\
& \times \pi^{-1} d^{2} \alpha,
\end{aligned}
$$

an integral whose value is given by the general expression (A2) as

$$
\operatorname{Tr}[F(\lambda) D(\xi)]=\frac{1}{(1-\lambda)} \exp \left(-\frac{(1+\lambda)|\xi|^{2}}{2(1-\lambda)}\right)
$$

According to Eq. (4.19) the coefficients $b_{n, m}$ of the symmetrically ordered power series for $F(\lambda)$ are given by the integrals

$$
\begin{array}{r}
b_{n, m}(\lambda)=\frac{\delta_{n, m}}{(n !)^{2}(1-\lambda)} \int \exp \left(-\frac{(1+\lambda)|\xi|^{2}}{2(1-\lambda)}\right) \\
\times\left(-|\xi|^{2}\right)^{n} \pi^{-1} d^{2} \xi,
\end{array}
$$

which converge whenever $F(\lambda)$ is bounded, i.e., for $|\lambda|<1$, and yield

$$
b_{n, m}(\lambda)=\frac{2 \delta_{n, m}}{n !(\lambda+1)}\left(2 \frac{\lambda-1}{\lambda+1}\right)^{n} .
$$

These coefficients are analytic functions of $\lambda$ and may be continued outside the region in which the integrals defining them converge. They all have a pole at $\lambda=-1$, where the operator $F(\lambda)$ is finite but not bounded. Using 
the symbol \{ \} once more to denote symmetric ordering, we may express the symmetrically ordered series for $F(\lambda)$ in the closed form

$$
F(\lambda)=\frac{2}{\lambda+1}\left\{\exp \left(2 a^{\dagger} a \frac{\lambda-1}{\lambda+1}\right)\right\}
$$

Turning now to the case of antinormal ordering, we find from Eq. (4.9) the result

$$
\begin{aligned}
d_{n, m}(\lambda) & =\frac{\delta_{n, m}}{(n !)^{2}(1-\lambda)} \int \exp \left(-\frac{\lambda|\xi|^{2}}{1-\lambda}\right)\left(-|\xi|^{2}\right)^{n} \pi^{-1} d^{2} \xi \\
& =\frac{\delta_{n, m}}{n ! \lambda}\left(1-\frac{1}{\lambda}\right)^{n} .
\end{aligned}
$$

These coefficients all have poles at $\lambda=0$ for which value the operator $F(\lambda)$ is the projection operator $|0\rangle\langle 0|$, which is both bounded and in the trace class. If we denote antinormal ordering by the symbol \{\}$_{A}$, then we may express the antinormally ordered series in the closed form

$$
F(\lambda)=\lambda^{-1}\left\{\exp \left[\left(1-\lambda^{-1}\right) a^{\dagger} a\right]\right\}_{A} .
$$

It may be shown ${ }^{2}$ that the antinormally ordered series for $F(\lambda)$ converges only for $\left|1-\lambda^{-1}\right|<1$ or equivalently for $\operatorname{Re} \lambda>\frac{1}{2}$.

\section{S-ORDERED POWER-SERIES EXPANSIONS}

Section IV contains some elementary observations about ordered power-series expansions. It was shown that normally ordered power series converge for virtually all operators of interest but that the coefficients of antinormally ordered expansions are singular even for some trace-class operators. In order to shed more light on this matter we introduce in this section a parametrized ordering convention according to which normal, symmetric, and antinormal ordering are distinguished by three distinct values of a continuous order parameter. By means of this convention we are able to vary the type of ordering in a continuous way from antinormal order to normal order and to see when the coefficients of the expansions become finite and when the expansions themselves become convergent. We show that for all bounded operators the coefficients are finite when the ordering is closer to normal than to antinormal ordering and that the series converge when the ordering is closer to normal than to symmetric ordering.

In introducing this ordering convention we do not suggest that the new types of orderings it defines have direct physical significance. It is intended instead as a useful device for understanding the problems associated with the three useful orderings - normal, symmetric, and antinormal ordering. Our main use of the ordering convention will be in connection with a parametrized integral representation which we introduce in Sec. VI.
It will be helpful there to observe the way the structure of this representation changes with variations of the order parameter.

We have seen in Eqs. (2.11)-(2.15) how to express the displacement operators in various ordered forms. Let us define the $s$-ordered displacement operator $D(\alpha, s)$ by the relation

$$
D(\alpha, s)=D(\alpha) e^{s|\alpha|^{2} / 2},
$$

where $s$ is a complex number. For the three discrete values of $s=+1,0,-1$, the operator $D(\alpha, s)$ can be written as an exponential which is, respectively, normally ordered

$$
D(\alpha, 1)=e^{\alpha a \dagger} e^{-\alpha^{*} a},
$$

symmetrically ordered

$$
D(\alpha, 0)=e^{\alpha a^{\dagger}-\alpha^{*} a},
$$

and antinormally ordered

$$
D(\alpha,-1)=e^{-\alpha^{*} a} e^{\alpha a^{\dagger}} .
$$

Proceeding as in our description of symmetric ordering, Eqs. (4.12)-(4.16), we define the s-ordered product $\left\{\left(a^{\dagger}\right)^{n} a^{m}\right\}_{s}$ by means of the Taylor series

$$
D(\alpha, s)=\sum_{n, m=0}^{\infty}\left\{\left(a^{\dagger}\right)^{n} a^{m}\right\}_{s}(n ! m !)^{-1} \alpha^{n}\left(-\alpha^{*}\right)^{m}
$$

or, equivalently, as the derivative

$$
\left\{\left(a^{\dagger}\right)^{n} a^{m}\right\}_{s}=\left.\frac{\partial^{n+m} D(\alpha, s)}{\partial \alpha^{n} \partial\left(-\alpha^{*}\right)^{m}}\right|_{\alpha=0}
$$

evaluated at $\alpha=0$

By applying this differential relation to the ordered exponentials (5.2)-(5.4), we find that the orderings specified by $s=+1,0,-1$ are, respectively, normal,

$$
\left\{\left(a^{\dagger}\right)^{n} a^{m}\right\}_{1}=\left(a^{\dagger}\right)^{n} a^{m}
$$

symmetric in the sense of Eqs. (4.12)-(4.16),

$$
\left\{\left(a^{\dagger}\right)^{n} a^{m}\right\}_{0}=\left\{\left(a^{\dagger}\right)^{n} a^{m}\right\},
$$

and antinormal,

$$
\left\{\left(a^{\dagger}\right)^{n} a^{m}\right\}_{-1}=a^{m}\left(a^{\dagger}\right)^{n} .
$$

The $s$-ordered products can be simply expressed in terms of normally ordered products. Thus, for example, by writing

$$
D(\alpha, s)=e^{(s-1)|\alpha|^{2} / 2} D(\alpha, 1),
$$

we find for the simplest nontrivial $s$-ordered product $\left\{a^{\dagger} a\right\}_{s}$

$$
\begin{aligned}
\left\{a^{\dagger} a\right\}_{s} & =\left.\frac{\partial^{2}}{\partial \alpha \partial\left(-\alpha^{*}\right)} e^{(s-1)|\alpha|^{2} / 2} e^{\alpha a \dagger} e^{-\alpha^{*} a}\right|_{\alpha=0} \\
& =\left.\frac{\partial}{\partial \alpha}\left[e^{\alpha a \dagger} a+\frac{1}{2}(1-s) \alpha e^{\alpha a^{\dagger}}\right]\right|_{\alpha=0} \\
& =a^{\dagger} a+\frac{1}{2}(1-s) .
\end{aligned}
$$


Similarly, for the $s$-ordered product $\left\{a^{\dagger} a^{2}\right\}_{s}$ we find

$$
\left\{a^{\dagger} a^{2}\right\}_{s}=a^{\dagger} a^{2}+(1-s) a .
$$

It is not difficult to express an arbitrary $s$-ordered product as a polynomial in the $t$-ordered products, where $t$ is also arbitrary. By writing

$$
D(\alpha, s)=e^{(s-t)|\alpha|^{2} / 2} D(\alpha, t)
$$

and differentiating, we find

$$
\left\{\left(a^{\dagger}\right)^{n} a^{m}\right\}_{s}=\sum_{k=0}^{(n, m)} k !\left(\begin{array}{l}
n \\
k
\end{array}\right)\left(\begin{array}{l}
m \\
k
\end{array}\right)\left(\frac{t-s}{2}\right)^{k}\left\{\left(a^{\dagger}\right)^{n-k} a^{m-k}\right\}_{t},
$$

where the symbol $(n, m)$ denotes the smaller of the integers $n$ and $m$ and where

$$
\left(\begin{array}{l}
n \\
k
\end{array}\right) \equiv r ![k !(r-k) !]^{-1}
$$

is a binomial coefficient. These relations may be put more succinctly in terms of the associated Laguerre polynomials. ${ }^{19}$ As we show in Appendix C, we have for $n \geq m$,

$$
\left\{\left(a^{\dagger}\right)^{n} a^{m}\right\}_{s}=m !\left(\frac{t-s}{2}\right)^{m}\left\{\left(a^{\dagger}\right)^{n-m} L_{m}^{(n-m)}\left(\frac{2 a^{\dagger} a}{s-t}\right)\right\}_{t}
$$

and for $m \geq n$

$$
\left\{\left(a^{\dagger}\right)^{n} a^{m}\right\}_{s}=n !\left(\frac{t-s}{2}\right)^{n}\left\{a^{m-n} L_{n}(m-n)\left(\frac{2 a^{\dagger} a}{s-t}\right)\right\}_{t},
$$

where the polynomials within the curly brackets are in $t$-ordered form.

We are now in a position to consider the expansion of a bounded operator $F$ as the $s$-ordered power series

$$
F=\sum_{n, m=0}^{\infty} f_{n, m}(s)\left\{\left(a^{\dagger}\right)^{n} a^{m}\right\}_{s},
$$

where the coefficients $f_{n, m}(s)$ are complex numbers. We may identify the coefficients $f_{n, m}(s)$ by substituting in the general expansion (3.22) the expression

$$
D^{-1}(\xi)=e^{-s|\xi|^{2} / 2} D(-\xi, s),
$$

so that we have

$$
F=\int \operatorname{Tr}[F D(\xi)] e^{-s|\xi|^{2 / 2}} D(-\xi, s) \pi^{-1} d^{2} \xi,
$$

which we may expand in powers of $a$ and $a^{\dagger}$ according to Eq. (5.5):

$$
\begin{aligned}
F=\sum_{n, m=0}^{\infty}\left\{\left(a^{\dagger}\right)^{n} a^{m}\right\}_{s}(n ! m !)^{-1} \int & \operatorname{Tr}[F D(\xi,-s)] \\
& \times(-\xi)^{n}\left(\xi^{*}\right)^{m} \pi^{-1} d^{2} \xi .
\end{aligned}
$$

In this way we obtain the coefficients $f_{n, m}(s)$ as the integrals

$$
\begin{aligned}
f_{n, m}(s)=(n ! m !)^{-1} \int \operatorname{Tr}[F D(\xi,- & -s)](-\xi)^{n} \\
& \times\left(\xi^{*}\right)^{m} \pi^{-1} d^{2} \xi .
\end{aligned}
$$

The parametrized relations (5.16)-(5.18) compactly express the previously derived results for normal, symmetric and antinormal ordering. We recover Eqs. (4.5), (4.19), and (4.9) by setting $s=+1,0$, and -1 , respectively.

By applying the inequality (3.9) and the relation (3.16) to the integral (5.18), we find that for all bounded operators $F$ the coefficients are finite when $\operatorname{Res}>0$ and are bounded by the quantities ${ }^{2}$

$$
\left|f_{n, m}(s)\right| \leq \frac{[(n+m) !]^{1 / 2}}{n ! m !} \frac{\|F\|}{[\operatorname{Re} s]^{(n+m+1) / 2}},
$$

where $\|F\|$ is the Hilbert-Schmidt norm (3.15). This inequality generalizes the upper bound (4.6), which was obtained for normal ordering, $s=1$, to those orderings specified by Res $>0$, i.e., to those which may be thought of as closer to normal than to antinormal order.

We recall from Sec. IV that the coefficients $f_{n, m}(0)$, corresponding to symmetric order, are singular for certain bounded operators. We may therefore say that the coefficients $f_{n, m}(s)$ are finite for all bounded operators $F$ if and only if Res $>0$.

Before turning to the question of the convergence of the series (5.15), we may note ${ }^{2}$ that if the operator $F$ is bounded then the coefficients $f_{n, m}(s)$ are all analytic functions of the order parameter $s$ throughout the halfplane Res $>0$. This analyticity is intuitively clear from the structure of the integrals (5.18) and from the squareintegrability of the weight functions $\operatorname{Tr}[F D(\xi)]$ belonging to bounded operators.

We defined a type of convergence that is suited to ordered power-series expansions in Sec. IV, Eqs. (4.20) and (4.21). We shall now show that for bounded operators $F$ the series (5.15) converges according to this definition when $\operatorname{Res}>\frac{1}{2}+\frac{1}{2}[\operatorname{Im} s]^{2}$.

The series (5.17) is the limit of the sequence of operators $F_{N}$,

$$
F_{N}=\sum_{n, m=0}^{N} f_{n, m}(s)\left\{\left(a^{\dagger}\right)^{n} a^{m}\right\}_{s},
$$

of which the coherent state matrix elements are

$$
\begin{aligned}
\left\langle\beta\left|F_{N}\right| \alpha\right\rangle= & \sum_{n, m=0}^{N} f_{n, m}(s)\left\langle\beta\left|\left\{\left(a^{\dagger}\right)^{n} a^{m}\right\}_{s}\right| \alpha\right\rangle \\
= & \left.\sum_{n, m=0}^{N} f_{n, m}(s) \frac{\partial^{n+m}}{\partial \zeta^{n} \partial\left(-\zeta^{*}\right)^{m}}\langle\beta|D(\zeta, s)| \alpha\rangle\right|_{\zeta=0} \\
= & \sum_{n, m=0}^{N} \int \operatorname{Tr}[F D(\xi,-s)] \frac{(-\xi)^{n}}{n !} \frac{\left(\xi^{*}\right)^{m}}{m !} \\
& \times\left(\left.\frac{\partial^{n+m}\langle\beta|D(\zeta, s)| \alpha\rangle}{\partial \zeta^{n} \partial\left(-\zeta^{*}\right)^{m}}\right|_{\zeta=0}\right) \pi^{-1} d^{2} \xi \ldots
\end{aligned}
$$


The sequence $\left\langle\beta\left|F_{N}\right| \alpha\right\rangle$ therefore, converges to $\langle\beta|F| \alpha\rangle$ when in this expression the limits of summation and integration may be interchanged. The relevant sequence of functions is

$$
\varphi_{N}(\xi)=\sum_{n, m=0}^{N} \frac{(-\xi)^{n}}{n !} \frac{\left(\xi^{*}\right)^{m}}{m !}\left(\left.\frac{\partial^{n+m}\langle\beta|D(\zeta, s)| \alpha\rangle}{\partial \zeta^{n} \partial\left(-\zeta^{*}\right)^{m}}\right|_{\zeta=0}\right)
$$

which converges to

$$
\begin{aligned}
\varphi(\xi) & =\langle\beta|D(-\xi, s)| \alpha\rangle \\
& =\langle\beta \mid \alpha\rangle \exp \left[\frac{1}{2}(s-1)|\xi|^{2}-\beta^{*} \xi+\alpha \xi^{*}\right] .
\end{aligned}
$$

It is not difficult to show that for all $N$ the functions $\varphi_{N}(\xi)$ are dominated in accordance with the inequality

$$
\begin{aligned}
\left|\varphi_{N}(\xi)\right| \leq M(\xi) \equiv|\langle\beta \mid \alpha\rangle| \exp \left[\frac{1}{2}|s-1||\xi|^{2}\right. \\
+(|\beta|+|\alpha|)|\xi|] .
\end{aligned}
$$

Thus, according to the Lebesgue dominated convergence theorem, ${ }^{17}$ the interchange of limits is permissible when the integral

$$
\int M(\xi)|\operatorname{Tr}[F D(\xi,-s)]| \pi^{-1} d^{2} \xi
$$

converges. Now for an arbitrary bounded operator $F$ this integral converges when Res $>|s-1|$, or, equivalently, when $s$ lies in the parabolic region

$$
\operatorname{Re} s>\frac{1}{2}+\frac{1}{2}[\operatorname{Im} s]^{2} \text {. }
$$

This condition is therefore sufficient for the series (5.15) to converge for every bounded operator $F$. In particular, all bounded operators possess convergent $s$-ordered power series for $s>\frac{1}{2}$, i.e., when the ordering is closer to normal than to symmetric.

The example considred in Sec. IV,

$$
F(\lambda)=\lambda^{a \dagger a},
$$

provides a simple illustration of some of the results that we have derived. By using Eq. (4.38), we find that the integrals (5.18) for the coefficients $f_{n, m}(s)$ assume the form

$$
\begin{aligned}
f_{n, m}(s)= & \frac{1}{n ! m !(1-\lambda)} \int \exp \left[\left(\frac{1-s}{2}+\frac{1}{\lambda-1}\right)|\xi|^{2}\right] \\
& \times(-\xi)^{n}\left(\xi^{*}\right)^{m} \frac{d^{2} \xi}{\pi} \\
= & \frac{\delta_{n, m}}{n !(\lambda-1)}\left(\frac{1-s}{2}+\frac{1}{\lambda-1}\right)^{-(n+1)},
\end{aligned}
$$

where we have used a series expansion of the formula (A2) to do the integral. The coefficients $f_{n, m}(s)$ are analytic functions of $s$ except for poles located at

$$
s=(\lambda+1) /(\lambda-1) .
$$

This relation is a familiar one; it maps the interior of the unit circle $|\lambda|<1$ onto the left half-plane $\operatorname{Res}<0$. Thus, the location of the singularities of the coefficients $f_{n, m}(s)$ ranges over the half-plane $\operatorname{Res}<0$ as the parameter $\lambda$ ranges over the region in which the operator $F(\lambda)$ is bounded. On summing the $s$-ordered power series for $F(\lambda)$, we obtain the expression

$F(\lambda)=\lambda^{a \dagger a}=\frac{2}{1+s+\lambda-s \lambda}\left\{\exp \left(\frac{2(\lambda-1) a^{\dagger} a}{1+s+\lambda-s \lambda}\right)\right\}_{s}$.

By examining the convergence of the integral (5.23), we find, taking $s$ real for simplicity, that the $s$-ordered power series for $F(\lambda)$ converges when

$$
s>1+\frac{\operatorname{Re} \lambda-1}{|\lambda-1|^{2}} .
$$

Thus, when $\lambda=0$ and $F(\lambda)=|0\rangle\langle 0|$, for example, the series (5.27) converges for $s>0$.

As a final illustration let us note that we may write the $s$-ordered displacement operator $D(\alpha, s)$ in the form

$$
\begin{aligned}
D(\alpha, s) & =e^{s|\alpha|^{2} / 2} D(\alpha) \\
& =\left\{\exp \left(\alpha a^{\dagger}-\alpha^{*} a\right)\right\}_{s} \\
& =\{D(\alpha)\}_{s},
\end{aligned}
$$

which justifies our calling it the $s$-ordered displacement operator.

\section{INTEGRAL EXPANSIONS FOR OPERATORS}

The representation of operators as integrals over the displacement operators is in many respects analogous to the representation of functions as Fourier integrals. The displacement-operator expansion

$$
F=\int e^{a \xi^{*}-a \dagger \xi} f(\xi) \pi^{-1} d^{2} \xi
$$

differs from the Fourier expansion

$$
g(\alpha)=\int e^{\alpha \xi^{*}-\alpha^{*} \xi} \varphi(\xi) \pi^{-1} d^{2} \xi
$$

because of the noncommutativity that distinguishes the variables $a$ and $a^{\dagger}$ from their counterparts $\alpha$ and $\alpha^{*}$. Distinctions in operator ordering lend an interesting structure to the Fourier representation of operators. We consider some elementary aspects of this structure in the present section. For each value of the order parameter $s$, we define a set of operators $T(\alpha, s)$ that forms a basis for an integral representation for arbitrary operators. Each of these representations has the desirable property that the associated weight function bears a particularly direct relationship to the operator being expanded.

These new representations are interesting from a number of standpoints. In particular, for the case of 
antinormal ordering the new expansion expresses operators as integrals over projection operators upon the coherent states. For the case in which the operator being expanded is a density operator, this expansion takes the form of the $P$ representation, ${ }^{7,23,24}$ which has been widely discussed in connection with problems in quantum optics. When the ordering is symmetric and the operator being expanded is a density operator, the weight function in the expansion is the one introduced by Wigner ${ }^{25}$ as a quantum-mechanical analog of the classical phase-space density function. We shall make a detailed application of the present formalism to the case of the density operator in the paper which follows.

Let us recall that according to Eq. (3.23) the trace of the product of any two bounded operators can be written as the integral

$$
\operatorname{Tr}[F G]=\int \operatorname{Tr}[F D(\xi)] \operatorname{Tr}[G D(-\xi)] \pi^{-1} d^{2} \xi,
$$

in which the functions $\operatorname{Tr}[F D(\xi)]$ and $\operatorname{Tr}[G D(-\xi)]$ are both square-integrable functions. By using the definition (5.1) of the operator $D(\xi, s)$,

$$
D(\xi, s)=e^{s|\xi|^{2} / 2} D(\xi),
$$

we may trivially reexpress this trace in the form

$$
\begin{array}{r}
\operatorname{Tr}[F G]=\int \operatorname{Tr}[F D(\xi,-s)] \operatorname{Tr}[G D(-\xi, s)] \\
\times \pi^{-1} d^{2} \xi .
\end{array}
$$

Now if both of the traces appearing in this integral are square-integrable functions, then they possess the complex Fourier transforms

$$
\begin{array}{r}
f(\alpha,-s)=\int \exp \left(\alpha \xi^{*}-\alpha^{*} \xi\right) \operatorname{Tr}[F D(\xi,-s)] \\
\times \pi^{-1} d^{2} \xi
\end{array}
$$

and

$$
g(\alpha, s)=\int \exp \left(\alpha \xi^{*}-\alpha^{*} \xi\right) \operatorname{Tr}[G D(\xi, s)] \pi^{-1} d^{2} \xi,
$$

which are also square-integrable, and we may apply the identity (3.7) to secure the result

$$
\operatorname{Tr}[F G]=\int f(\alpha,-s) g(\alpha, s) \pi^{-1} d^{2} \xi .
$$

The condition that the traces $\operatorname{Tr}[F D(\xi,-s)]$ and $\operatorname{Tr}[G D(\xi, s)]$ both be square-integrable is clearly satisfied for all bounded operators $F$ and $G$ when $\operatorname{Re} s=0$. The relation (6.5) is consequently for $\operatorname{Re} s=0$ an identity holding for all bounded operators. It is likewise true that for every value of $s$ one of the traces,

${ }^{23}$ R. J. Glauber, Phys. Rev. Letters 10, 84 (1963).

${ }^{24}$ E. C. G. Sudarshan, Phys. Rev. Letters 10, 277 (1963).

${ }^{25}$ E. P. Wigner, Phys. Rev. 40, 749 (1932).
$\operatorname{Tr}[F D(\xi,-s)]$ or $\operatorname{Tr}[G D(-\xi, s)]$, will be squareintegrable given that both operators are bounded. Because these traces depend upon the parameter $s$ through the factors $\exp \left( \pm \frac{1}{2} s|\xi|^{2}\right)$, their mathematical properties depend primarily upon the real part of $s$. We shall discuss the occurrence of singularities in Eqs. (6.3)-(6.5) at a later point; we may infer for the present that for every two bounded operators $F$ and $G$ there will, in general, be a strip $x_{1}<\operatorname{Res}<x_{2}$ about the imaginary axis of the $s$ plane in which Eqs. (6.3)-(6.5) are valid.

Let us define the operator $T(\alpha, s)$ as the complex Fourier transform of the $s$-ordered displacement operator $D(\xi, s)$,

$$
T(\alpha, s)=\int e^{\alpha \xi^{*}-\alpha^{*} \xi} D(\xi, s) \pi^{-1} d^{2} \xi .
$$

This notation allows us to express Eqs. (6.3)-(6.5) in the more compact form

$$
\begin{aligned}
f(\alpha,-s) & =\operatorname{Tr}[F T(\alpha,-s)], \\
g(\alpha, s) & =\operatorname{Tr}[G T(\alpha, s)],
\end{aligned}
$$

and

$\operatorname{Tr}[F G]=\int \operatorname{Tr}[F T(\alpha,-s)] \operatorname{Tr}[G T(\alpha, s)] \pi^{-1} d^{2} \alpha$.

When the operator $G$ in Eq. (6.9) is taken to be the outer product of two states $|\psi\rangle$ and $|\varphi\rangle$, we have

$$
\begin{aligned}
\langle\varphi|F| \psi\rangle & =\int \operatorname{Tr}[F T(\alpha,-s)]\langle\varphi|T(\alpha, s)| \psi\rangle \pi^{-1} d^{2} \alpha \\
& =\int f(\alpha,-s)\langle\varphi|T(\alpha, s)| \psi\rangle \pi^{-1} d^{2} \alpha
\end{aligned}
$$

If for some value of $s$ this relation holds for all normalizable states $|\varphi\rangle$ and $|\psi\rangle$, then we may say that the operator $F$ possesses the representation

$$
\begin{aligned}
F & =\int f(\alpha,-s) T(\alpha, s) \pi^{-1} d^{2} \alpha \\
& =\int \operatorname{Tr}[F T(\alpha,-s)] T(\alpha, s) \pi^{-1} d^{2} \alpha
\end{aligned}
$$

for that value of $s$.

There are, of course, many senses in which an operator $F$ can be said to possess this representation for a particular value of $s$. We might, for example, require only that Eq. (6.10) hold for some complete set of states. A stronger condition would be that Eq. (6.9) be true for all bounded operators $G$. Alternatively, by interpreting Eqs. (6.9)-(6.11) in terms of limiting processes or in terms of generalized functions, ${ }^{2}$ it is possible to set down conditions that are so broad as to include all bounded operators $F$ and all values of $s$, 
By using Eqs. (5.29) and (3.3) we may express the operator $T(\alpha, s)$ in the suggestive forms

$$
\begin{aligned}
T(\alpha, s) & =\left\{\int \exp \left[(\alpha-a) \xi^{*}-\left(\alpha^{*}-a^{\dagger}\right) \xi\right] \pi^{-1} d^{2} \xi\right\}_{s} \\
& =\pi\left\{\delta^{(2)}(\alpha-a)\right\}_{s} .
\end{aligned}
$$

In terms of this notation we may write the representation (6.11) in the form

$$
F=\int f(\alpha,-s)\left\{\delta^{(2)}(\alpha-a)\right\}_{s} d^{2} \alpha .
$$

The classical analog of this representation is therefore the trivial identity

$$
g(\alpha)=\int g\left(\alpha^{\prime}\right) \delta^{(2)}\left(\alpha-\alpha^{\prime}\right) d^{2} \alpha^{\prime}
$$

in which the relationship between the function being expanded and its weight function is one of identity. The considerably more interesting structure of the representation (6.14) arises, of course, from the noncommutativity of the operators $a$ and $a^{\dagger}$.

On the basis of the analogy between Eqs. (6.14) and (6.15), we may reasonably expect to find a close relationship between the weight function $f(\alpha,-s)$ and the operator $F$ in Eq. (6.14). We shall describe this relationship and other properties of these representations in Sec. VII. The remainder of the present section is devoted to the properties of the operators $T(\alpha, s)$.

By using the definition (6.6) to form the Hermitian adjoint

$$
T^{\dagger}(\alpha, s)=\int D\left(-\xi, s^{*}\right) \exp \left(\alpha^{*} \xi-\alpha \xi^{*}\right) \pi^{-1} d^{2} \xi
$$

and replacing $\xi$ by $-\xi$, we find

$$
T^{\dagger}(\alpha, s)=T\left(\alpha, s^{*}\right) .
$$

Thus for real values of the order parameter $s$, the operator $T(\alpha, s)$ is Hermitian,

$$
T(\alpha, s)=T^{\dagger}(\alpha, s) \text { for } s \text { real. }
$$

From the multiplication law (2.19), we find

$$
D(\alpha) D(\xi, s) D^{-1}(\alpha)=D(\xi, s) \exp \left(\alpha \xi^{*}-\alpha^{*} \xi\right),
$$

which, when substituted into the definition (6.6), yields

$$
\begin{aligned}
T(\alpha, s) & =\int D(\alpha) D(\xi, s) D^{-1}(\alpha) \pi^{-1} d^{2} \xi \\
& =D(\alpha) T(0, s) D^{-1}(\alpha) .
\end{aligned}
$$

Thus, the $\alpha$ dependence of the operator $T(\alpha, s)$ is governed by the unitary transformation induced by the operator $D(\alpha)$. On using Eq. (2.19) once again we find

$$
T(\alpha, s)=D(\alpha-\beta) T(\beta, s) D^{-1}(\alpha-\beta) .
$$

To find the $s$ dependence of the operator $T(\alpha, s)$ we note that the operator $T(0, s)$ is defined by Eq. (6.6) as the integral

$$
T(0, s)=\int D^{-1}(\xi) e^{s|\xi|^{2} / 2} \pi^{-1} d^{2} \xi .
$$

This is a displacement operator expansion of the form of Eq. (3.22) with weight function

$$
\operatorname{Tr}[T(0, s) D(\xi)]=e^{s|\xi|^{2} / 2} .
$$

We encountered a similar weight function in our discussion of the operator $F(\lambda)=\lambda^{a^{\dagger} a}$ and, if we compare Eqs. (6.20) and (6.21) with Eq. (4.38) and make the identification

$$
s=\frac{\lambda+1}{\lambda-1} \text { or } \lambda=\frac{s+1}{s-1},
$$

then, since the correspondence $F \leftrightarrow \operatorname{Tr}[F D(\xi)]$ is oneto-one, we secure the result

$$
T(0, s)=\frac{2}{1-s}\left(\frac{s+1}{s-1}\right)^{a \ddagger a} .
$$

By using Eq. (6.18) and the displacement property (2.17) and (2.18) of the unitary operators $D(\alpha)$, we obtain for the operator $T(\alpha, s)$ the following expressions:

$$
\begin{aligned}
& T(\alpha, s)=\frac{2}{1-s} D(\alpha)\left(\frac{s+1}{s-1}\right)^{a \dagger a} D^{-1}(\alpha) \\
& =\frac{2}{1-s}\left(\frac{s+1}{s-1}\right)^{\left(a \dagger-\alpha^{*}\right)(a-\alpha)} \\
& =\frac{2}{1-s} \exp \left[\left(a^{\dagger}-\alpha^{*}\right)(a-\alpha) \ln \left(\frac{s+1}{s-1}\right)\right] \text {. }
\end{aligned}
$$

The expansion of $T(\alpha, s)$ in terms of the eigenstates $|n\rangle$ of the operator $a^{\dagger} a$ is, from Eq. (6.23),

$$
T(\alpha, s)=\frac{2}{1-s} \sum_{n=0}^{\infty} D(\alpha)|n\rangle\left(\frac{s+1}{s-1}\right)^{n}\langle n| D^{\dagger}(\alpha) .
$$

The states $D(\alpha)|n\rangle$ thus form a complete orthornormal set of eigenstates of the operator $T(\alpha, s)$,

$$
T(\alpha, s) D(\alpha)|n\rangle=\frac{2}{1-s}\left(\frac{s+1}{s-1}\right)^{n} D(\alpha)|n\rangle,
$$

with eigenvalues

$$
e_{n}(s)=\frac{2}{1-s}\left(\frac{s+1}{s-1}\right)^{n}
$$

which are independent of $\alpha$.

We note in particular that for all values of $\alpha$ all the eigenvalues $e_{n}(s)$ of the operator $T(\alpha, s)$ are infinite at $s=1$. At $s=-1$, on the other hand, the series (6.26) 
terminates after the first term and the operator $T(\alpha,-1)$ is simply the projection operator on the coherent state $|\alpha\rangle=D(\alpha)|0\rangle$

$$
T(\alpha,-1)=|\alpha\rangle\langle\alpha| \text {. }
$$

Let us now consider to what classes of operators the operator $T(\alpha, s)$ belongs for different values of $s$. The three norms that we defined in Sec. III are invariant under unitary transformations and do not, therefore, for the case of the operator $T(\alpha, s)$ depend upon the parameter $\alpha$. By using Eqs. (3.13)-(3.15) and (6.21), we may express the Hilbert-Schmidt norm $\|T(\alpha, s)\|$ in terms of the integral

$$
\begin{aligned}
\|T(\alpha, s)\|^{2} & =\int\left|e^{s|\xi|^{2} / 2}\right|^{2} \pi^{-1} d^{2} \xi \\
& =\int e^{\text {Res }|\xi|^{2}} \pi^{-1} d^{2} \xi
\end{aligned}
$$

so that we have

$$
\|T(\alpha, s)\|=1 /(-\operatorname{Res})^{1 / 2} \quad \text { for } \operatorname{Re} s<0 .
$$

The operator $T(\alpha, s)$ is therefore a bounded operator only for $\operatorname{Re} s<0$. When $\operatorname{Re} s \leq 0$ the operator $T(\alpha, s)$ is a finite operator, and by using Eq. (6.26) we find for its norm $\|T(\alpha, s)\|_{1}$, defined by Eq. (3.33), the value

$$
\|T(\alpha, s)\|_{1}=\left|\frac{2}{1-s}\right| \quad \text { for } \quad \operatorname{Re} s \leq 0 .
$$

For $\operatorname{Res}<0$ the operator $T(\alpha, s)$ is not only bounded but also in the trace class. By using Eq. (6.26) we obtain for its trace class norm (3.37) the series

$$
\begin{aligned}
\|T(\alpha, s)\|_{2} & =\frac{2}{|1-s|} \sum_{n=0}^{\infty}\left|\frac{s+1}{s-1}\right|^{n} \\
& =2 /(|1-s|-|1+s|) \text { for } \operatorname{Res}<0 .
\end{aligned}
$$

Thus, for $\operatorname{Res}<0$ the operator $T(\alpha, s)$ is a member of all three classes of operators, for $\operatorname{Re} s>0$ it is in none of them, and on the line Res $=0$ it is in only the largest class, the class of finite operators.

By referring to the relation (4.35) between the operator $F(\lambda)$ and its normally ordered form we find

$$
\begin{aligned}
T(\alpha, s)= & \frac{2}{1-s}: \exp \left[\left(\frac{2}{s-1}\right)\left(\alpha^{\dagger}-\alpha^{*}\right)(a-\alpha)\right]: \\
= & \exp \left(-\frac{2|\alpha|^{2}}{1-s}\right) \exp \left(\frac{2 \alpha a^{\dagger}}{1-s}\right) T(0, s) \\
& \times \exp \left(\frac{2 \alpha^{*} a}{1-s}\right),
\end{aligned}
$$

where the colons denote normal ordering. From Eq. (6.33) there follow easily for the coherent-state matrix elements the relations

$$
\begin{aligned}
\langle\beta|T(\alpha, s)| \gamma\rangle= & \frac{2\langle\beta \mid \gamma\rangle}{1-s} \\
& \times \exp \left[\left(\frac{2}{s-1}\right)\left(\beta^{*}-\alpha^{*}\right)(\gamma-\alpha)\right]
\end{aligned}
$$

and

$$
\langle\beta|T(\alpha, s)| \beta\rangle=\frac{2}{1-s} \exp \left(-\frac{2|\alpha-\beta|^{2}}{1-s}\right),
$$

from which, by taking the limit as $s$ approaches unity from smaller real values, we find

$$
\langle\beta|T(\alpha, 1)| \beta\rangle=\pi \delta^{(2)}(\alpha-\beta) .
$$

By using Eqs. (6.36) and (2.28), we may express the trace of the operator $T(\alpha, s)$ as the integral

$$
\begin{aligned}
\operatorname{Tr}[T(\alpha, s)] & =\int\langle\beta|T(\alpha, s)| \beta\rangle \pi^{-1} d^{2} \beta \\
& =\frac{2}{1-s} \int \exp \left(-\frac{2|\alpha-\beta|^{2}}{1-s}\right) \frac{d^{2} \beta}{\pi} \\
& =1 \text { for } \operatorname{Res}<1 .
\end{aligned}
$$

That the operator $T(\alpha, s)$ is of unit trace can also be seen by summing its eigenvalues $e_{n}(s)$ which are given by Eq. (6.28). The procedure given above is a rearrangement of the series $\Sigma e_{n}(s)$ which for Res $\geq 0$ lies outside its radius of convergence but can still be summed to unity.

Another trace which will be useful in what follows is $\operatorname{Tr}[T(\alpha, s) T(\beta, t)]$. By using the definition (6.6) and the orthogonality rule (3.11) we find that

$$
\begin{aligned}
& \operatorname{Tr}[T(\alpha, s) T(\beta, t)]= \int \operatorname{Tr}[D(\xi, s) D(\zeta, t)] \\
& \times \exp \left(\alpha \xi^{*}-\alpha^{*} \xi+\beta \zeta^{*}-\beta^{*} \zeta\right) \\
& \times \pi^{-2} d^{2} \xi d^{2} \zeta \\
&= \\
&=\frac{-2}{s+t} \exp \left(\frac{2|\alpha-\beta|^{2}}{s+t}\right) \\
& \text { for } \operatorname{Re}(s+t)<0,
\end{aligned}
$$

where we have used the formula (A2) to do the integral, which converges only for $\operatorname{Re}(s+t)<0$. By letting $t$ approach $-s$ from below we find

$$
\operatorname{Tr}[T(\alpha, s) T(\beta,-s)]=\pi \delta^{(2)}(\alpha-\beta),
$$

which is the counterpart for the operators $T(\alpha, s)$ to the orthogonality rule (3.11) for the operators $D(\xi, s)$. 
If we now apply the expansion (6.11) to the operator $T(\alpha, s)$, we find, using the trace relation (6.39),

$$
\begin{aligned}
& T(\alpha, s)=\int T(\beta, t) \operatorname{Tr}[T(\alpha, s) T(\beta,-t)] \pi^{-1} d^{2} \beta \\
&=\frac{2}{t-s} \int \exp \left(\frac{-2|\alpha-\beta|^{2}}{t-s}\right) T(\beta, t) \frac{d^{2} \beta}{\pi} \\
& \text { for } \operatorname{Re} t>\operatorname{Res} .
\end{aligned}
$$

This Gaussian convolution is the complex Fourier transform of the product $\exp \left[\frac{1}{2}(s-t)|\xi|^{2}\right] D(\xi, t)$. The differential form of this integral relation can be found by differentiating both sides of Eq. (6.6); it is

$$
\frac{\partial T(\alpha, s)}{\partial s}=-\frac{1}{2} \frac{\partial^{2} T(\alpha, s)}{\partial \alpha \partial \alpha^{*}}
$$

By further differentiation of Eq. (6.6), we obtain the relations

$$
\left.\frac{\partial^{n+m} T(\alpha, s)}{\partial \alpha^{n} \partial\left(\alpha^{*}\right)^{m}}\right|_{\alpha=0}=\int D(\xi, s)\left(\xi^{*}\right)^{n}(-\xi)^{m} \frac{d^{2} \xi}{\pi} .
$$

If we use Eqs. (3.4) and (3.5) to invert Eq. (6.6), we find that

$$
D(\xi, s)=\int T(\alpha, s) \exp \left(\xi \alpha^{*}-\xi^{*} \alpha\right) \pi^{-1} d^{2} \alpha
$$

and, by expanding both sides in powers of $\xi$ and $\xi^{*}$ and using the definition (5.6), we may express the $s$-ordered products as the integrals

$$
\left\{\left(a^{\dagger}\right)^{n} a^{m}\right\}_{s}=\int T(\alpha, s)\left(\alpha^{*}\right)^{n} \alpha^{m} \pi^{-1} d^{2} \alpha .
$$

The fact that under integration the operator $T(\alpha, s)$ turns the monomial $\left(\alpha^{*}\right)^{n} \alpha^{m}$ into the $s$-ordered product $\left\{\left(a^{\dagger}\right)^{n} a^{m}\right\}_{s}$ illustrates again the sense in which it is an $s$-ordered operator analog of the $\delta$ function. As in the use of singular functions, some discrimination is called for in the application of this relation, particularly for Res $>0$, where $T(\alpha, s)$ is not a finite operator and at $s=1$ where it is explicitly singular.

When $n=m=0$ Eq. (6.45) becomes

$$
1=\int T(\alpha, s) \pi^{-1} d^{2} \alpha
$$

which for $s=-1$ is the completeness relation (2.27)

$$
1=\int|\alpha\rangle\langle\alpha| \pi^{-1} d^{2} \alpha
$$

for the coherent states.
By applying the orthogonality rule for the $T$ operators (6.40) to Eq. (6.45), we obtain the relation

$$
\begin{aligned}
& \operatorname{Tr}\left[\left\{\left(a^{\dagger}\right)^{n} a^{m}\right\}_{s} T(\alpha,-s)\right] \\
& =\int \operatorname{Tr}[T(\beta, s) T(\alpha,-s)]\left(\beta^{*}\right)^{n} \beta^{m} \pi^{-1} d^{2} \beta \\
& =\int\left(\beta^{*}\right)^{n} \beta^{m} \delta^{(2)}(\alpha-\beta) d^{2} \beta \\
& =\left(\alpha^{*}\right)^{n} \alpha^{m},
\end{aligned}
$$

which is the inverse of the relation (6.45).

From the relation (6.18) with $\alpha$ infinitesimal we find that

$$
d T(\alpha, s)=\left[a^{\dagger} d \alpha-a d \alpha^{*}, T(\alpha, s)\right]
$$

or, equivalently,

and

$$
\partial T(\alpha, s) / \partial \alpha=\left[a^{\dagger}, T(\alpha, s)\right]
$$

$$
\partial T(\alpha, s) / \partial \alpha^{*}=-[a, T(\alpha, s)],
$$

which are the complex Fourier transforms of the commutation relations implicit in Eqs. (2.17) and (2.18).

From Eq. (6.36) by a process of differentiation we may obtain the matrix elements $\langle n|T(\alpha, s)| m\rangle$. As we show in Appendix D, the result of that calculation is

$$
\begin{aligned}
\langle n|T(\alpha, s)| m\rangle= & \left(\frac{n !}{m !}\right)^{1 / 2}\left(\frac{2}{1-s}\right)^{m-n+1}\left(\frac{s+1}{s-1}\right)^{n}\left(\alpha^{*}\right)^{m-n} \\
& \times \exp \left(-\frac{2|\alpha|^{2}}{1-s}\right) L_{n}^{(m-n)}\left(\frac{4|\alpha|^{2}}{1-s^{2}}\right),
\end{aligned}
$$

where $L_{n}{ }^{(m)}(x)$ is an associated Laguerre polynomial. ${ }^{19}$

\section{CORRESPONDENCES BETWEEN OPERATORS AND FUNCTIONS}

In Sec. VI we introduced the operators $T(\alpha, s)$ which form, for each value of the order parameter $s$, a basis for the expansion of operators as weighted integrals. In this section we show that each of these integral representations maintains a close relationship between the operator being expanded and its weight function. We show that the mathematical properties of the weight functions change substantially as the order parameter is varied from $s=-1$, antinormal order, to $s=1$, normal order.

We have seen that, at least in the vicinity of the line Res $=0$, every bounded operator $F$ possesses the representation

$$
F=\int f(\alpha,-s) T(\alpha, s) \pi^{-1} d^{2} \alpha
$$

where the weight function $f(\alpha,-s)$ is given by the trace

$$
f(\alpha,-s)=\operatorname{Tr}[F T(\alpha,-s)] .
$$


Let us note that when the expansion is in terms of the operators $T(\alpha, s)$ the parameter $s$ appears in the weight function $f(\alpha,-s)$ with a minus sign.

We now observe that the trace (7.2) is the unique weight function for the operator $F$ in the expansion (7.1). For if the operator $F$ and the function $g(\alpha)$ stand in the relationship

$$
F=\int g\left(\alpha^{\prime}\right) T\left(\alpha^{\prime}, s\right) \pi^{-1} d^{2} \alpha^{\prime},
$$

then by using the trace relation (6.40) we find that

$$
\begin{aligned}
\operatorname{Tr}[F T(\alpha,-s)] & =\int g\left(\alpha^{\prime}\right) \operatorname{Tr}\left[T\left(\alpha^{\prime}, s\right) T(\alpha,-s)\right] \pi^{-1} d^{2} \alpha^{\prime} \\
& =\int g\left(\alpha^{\prime}\right) \delta^{(2)}\left(\alpha-\alpha^{\prime}\right) d^{2} \alpha^{\prime} \\
& =g(\alpha) .
\end{aligned}
$$

We may regard Eqs. (7.1) and (7.2) as defining, for every value of $s$, a correspondence $F \leftrightarrow f(\alpha,-s)$ between operators and their weight functions. Since the weight function corresponding to a given operator is unique, these correspondences are one-to-one.

It is in terms of power-series expansions that the correspondences, which we have just introduced, take their simplest form. If we assume that the operator $F$ possesses the $s$-ordered power-series expansion

$$
F=\sum_{n, m=0}^{\infty} f_{n, m}(s)\left\{\left(a^{\dagger}\right)^{n} a^{m}\right\}_{s},
$$

where the coefficients are given by Eq. (5.18), then by using Eq. (6.47) we may secure for the weight function $f(\alpha,-s)$ the power-series expansion

$$
\begin{aligned}
f(\alpha,-s) & =\operatorname{Tr}[F T(\alpha,-s)] \\
& =\sum_{n, m=0}^{\infty} f_{n, m}(s) \operatorname{Tr}\left[\left\{\left(a^{\dagger}\right)^{n} a^{m}\right\}_{s} T(\alpha,-s)\right] \\
& =\sum_{n, m=0}^{\infty} f_{n, m}(s)\left(\alpha^{*}\right)^{n} \alpha^{m},
\end{aligned}
$$

with the same coefficients $f_{n, m}(s)$. Conversely, if we assume for the function $f(\alpha,-s)$ this series expansion, then by using Eq. (6.45) we may obtain the operator $F$ in the form

$$
\begin{aligned}
F & =\int f(\alpha,-s) T(\alpha, s) \pi^{-1} d^{2} \alpha \\
& =\sum_{n, m=0}^{\infty} f_{n, m}(s) \int\left(\alpha^{*}\right)^{n} \alpha^{m} T(\alpha, s) \pi^{-1} d^{2} \alpha \\
& =\sum_{n, m=0}^{\infty} f_{n, m}(s)\left\{\left(a^{\dagger}\right)^{n} a^{m}\right\}_{s} .
\end{aligned}
$$

Thus, for every complex number $s$ the operator-function correspondence

$$
F \leftrightarrow f(\alpha,-s)=\operatorname{Tr}[F T(\alpha,-s)]
$$

involves simply the interchange

$$
\left\{\left(a^{\dagger}\right)^{n} a^{m}\right\}_{s} \leftrightarrow\left(\alpha^{*}\right)^{n} \alpha^{m}
$$

which is affected by the reciprocal relations (6.45) and (6.47).

We shall refer to this correspondence as the correspondence $C(s)$ or, when it is clear which value of the order parameter is meant, as the $s$-correspondence. Another way of expressing the correspondence $C(s)$ is to say that the weight function $f(\alpha,-s)$ is a generating function for the coefficients $f_{n, m}(s)$ of the $s$-ordered power-series expansion for $F$ according to the rule

$$
\left.\frac{\partial^{n+m} f(\alpha,-s)}{\partial \alpha^{n} \partial\left(\alpha^{*}\right)^{m}}\right|_{\alpha=0}=n ! m ! f_{n, m}(s) .
$$

The consistency of this prescription for the coefficients $f_{n, m}(s)$ with the earlier one (5.18) follows from Eq. (6.43). Two simple examples of the correspondence $C(s)$ are

$$
D(\xi, s) \leftrightarrow e^{\xi \alpha^{*}-\xi^{*} \alpha}
$$

and

$$
T(\xi, s) \leftrightarrow \pi \delta^{(2)}(\alpha-\xi)
$$

There are two ways in which singularities can occur in the representation (7.1). For $\operatorname{Re} s>0$ the operator $T(\alpha, s)$ is not a finite operator and when we form a matrix element $\langle\varphi|F| \psi\rangle$ of the representation (7.1) singularities can arise in the function $\langle\varphi|T(\alpha, s)| \psi\rangle$ which appears in the integral (6.10). On the other hand, when Res $<0$ the weight function $f(\alpha,-s)$ can become singular because the operator $T(\alpha,-s)$ in the trace $f(\alpha,-s)=\operatorname{Tr}[F T(\alpha,-s)]$ is not a finite operator. Such behavior on the part of the weight function $f(\alpha,-s)$ for $\operatorname{Re}<<0$ is of course related via Eq. (7.10) to singularities in the coefficients $f_{n, m}(s)$, the occurrence of which we discussed in Secs. IV and V.

The trace relation

$$
\begin{aligned}
\operatorname{Tr}[F G] & =\int f(\alpha,-s) g(\alpha, s) \pi^{-1} d^{2} \alpha \\
& =\int \operatorname{Tr}[F T(\alpha,-s)] \operatorname{Tr}[G T(\alpha, s)] \pi^{-1} d^{2} \alpha
\end{aligned}
$$

illustrates the need for caution in departing far from the line $\operatorname{Re} s=0$ since both operators $T(\alpha, s)$ and $T(\alpha,-s)$ appear explicitly. We note that the functions $f(\alpha,-s)$ and $g(\alpha, s)$ are associated with the operators $F$ and $G$ by the correspondences $C(s)$ and $C(-s)$, respectively. 
Only for $s=0$, symmetric order, do the two correspondences coincide.

The correspondence $C(1)$ associated with normal ordering, $s=1$, is particularly simple. As we have shown in Sec. IV, virtually every operator $F$ possesses a convergent normally ordered power-series expansion

$$
F=\sum_{n, m=0}^{\infty} f_{n, m}(1)\left(a^{\dagger}\right)^{n} a^{m} .
$$

The corresponding function

$$
f(\alpha,-1)=\operatorname{Tr}[F T(\alpha,-1)]
$$

is, according to Eq. (6.29), just the diagonal coherentstate matrix element

$$
f(\alpha,-1)=\langle\alpha|F| \alpha\rangle
$$

Although the correspondence $C(1)$ is well defined for an extremely broad class of operators, at $s=1$ the integral expansion (7.1),

$$
F=\int\langle\alpha|F| \alpha\rangle T(\alpha, 1) \pi^{-1} d^{2} \alpha,
$$

is of decidedly less generality since it involves the operator $T(\alpha, 1)$, all of whose eigenvalues are infinite, as shown by Eq. (6.28). In fact, it may be shown ${ }^{2}$ that for no states $|\psi\rangle$ and $|\varphi\rangle$ is the matrix element $\langle\varphi|T(\alpha, 1)| \psi\rangle$ a square-integrable function of $\alpha$.

For the case of antinormal ordering, $s=-1$, the correspondence $C(-1)$ associates with an operator $F$ the function

$$
f(\alpha, 1)=\operatorname{Tr}[F T(\alpha, 1)]
$$

which is clearly not in general free of singularities. These singularities are intimately related to those which occur in the coefficients of the antinormally ordered expansion

$$
F=\sum_{n, m=0}^{\infty} f_{n, m}(-1) a^{m}\left(a^{\dagger}\right)^{n} .
$$

For only when the function $f(\alpha, 1)$ is infinitely differentiable at $\alpha=0$ are the coefficients $f_{n, m}(-1)$ finite, as is shown by Eq. (7.10). When, however, the function $f(\alpha, 1)$ is well behaved, then according to Eq. (6.29) the expansion (7.1) assumes the simple form

$$
\begin{aligned}
F & =\int f(\alpha, 1) T(\alpha,-1) \pi^{-1} d^{2} \alpha \\
& =\int f(\alpha, 1)|\alpha\rangle\langle\alpha| \pi^{-1} d^{2} \alpha
\end{aligned}
$$

For the case of the density operator $\rho$, this is the $P$ representation ${ }^{23,7,24}$

$$
\rho=\int P(\alpha)|\alpha\rangle\langle\alpha| d^{2} \alpha
$$

where the weight function $P(\alpha)$ is given $b^{2,3}$

$$
P(\alpha)=\pi^{-1} \operatorname{Tr}[\rho T(\alpha, 1)] .
$$

Applications of the present results to the representation of density operators and to $P$ representation in particular are discussed in the following paper.

There are no serious problems in the correspondence $C(0)$ associated with symmetric order, $s=0$. The function $f(\alpha, 0)$ associated with the operator $F$,

$$
f(\alpha, 0)=\operatorname{Tr}[F T(\alpha, 0)],
$$

is the weight function for the expansion

$$
F=\int f(\alpha, 0) T(\alpha, 0) \pi^{-1} d^{2} \alpha .
$$

The operator $T(\alpha, 0)$ appearing in these relations is finite, though not bounded; and, as we observed in Sec. VI, the function $f(\alpha, 0)$ is in $L_{2}$ (i.e., is square-integrable) when the operator $F$ is bounded.

We recall, from Eqs. (3.12)-(3.16) that the correspondence $F \leftrightarrow \operatorname{Tr}[F D(\xi)]$ is one-to-one between $L_{2}$ and the class of all bounded operators and, from Eqs. (3.4)-(3.6), that the Fourier transform maps $L_{2}$ onto $L_{2}$ in a one to one fashion. Thus, since we defined the weight function $f(\alpha, 0)$ as the complex Fourier transform (6.3) of the function $\operatorname{Tr}[F D(\xi)]$, it follows that the symmetric correspondence $C(0)$ maps $L_{2}$ onto the class of all bounded operators in a one-to-one fashion. The correspondence $C(0)$ is also, in view of Eqs. (3.6) and (3.16), norm-preserving in that the $L_{2}$ norm (3.8) of the weight function $f(\alpha, 0)$ is equal to the Hilbert-Schmidt norm (3.15) of the associated operator $F$; i.e., we have

$$
\|f(\alpha, 0)\|=\|F\| \text {. }
$$

In terms of power-series expansions, the symmetric correspondence $C(0)$ associates the operator

$$
F=\sum_{n, m=0}^{\infty} f_{n, m}(0)\left\{\left(a^{\dagger}\right)^{n} a^{m}\right\}_{0}
$$

with the function

$$
f(\alpha, 0)=\sum_{n, m=0}^{\infty} f_{n, m}(0)\left(\alpha^{*}\right)^{n} \alpha^{m},
$$

where, as we have seen in Secs. IV and V, the coefficients $f_{n, m}(0)$ are finite for most though not all bounded operators $F$.

The correspondence $C(s)$ can easily be written in terms of complex Fourier transforms. By using Eqs. (3.7) and (6.6), we see that the correspondence $C(s)$ associates the operator

$$
\begin{aligned}
F & =\int f(\alpha,-s) T(\alpha, s) \pi^{-1} d^{2} \alpha \\
& =\int g(\xi,-s) D(-\xi, s) \pi^{-1} d^{2} \xi
\end{aligned}
$$


with the function

$$
\begin{aligned}
f(\alpha,-s) & =\operatorname{Tr}[F T(\alpha,-s)] \\
& =\int g(\xi,-s) \exp \left(\alpha \xi^{*}-\alpha^{*} \xi\right) \pi^{-1} d^{2} \xi
\end{aligned}
$$

By setting $s=0$ and using Eq. (2.1) and (2.2) to write these relations in real rather than complex notation, we may express the symmetric correspondence $C(0)$ in the form in which it was introduced by Weyl ${ }^{6}$ :

$$
\begin{gathered}
F=\int g\left(q^{\prime}, p^{\prime}\right) e^{-i\left(q p^{\prime}-p q^{\prime}\right)}(2 \pi)^{-1} d q^{\prime} d p^{\prime}, \\
f(x, y)=\int g\left(q^{\prime}, p^{\prime}\right) e^{-i\left(x p^{\prime}-y q^{\prime}\right)}(2 \pi)^{-1} d q^{\prime} d p^{\prime},
\end{gathered}
$$

where we have set $\hbar=1$.

It is a straightforward matter to derive a number of the properties of the weight functions $f(\alpha,-s)$ by using the analysis of the operators $T(\alpha, s)$ which was presented in Sec. VI. To avoid unnecessary minus signs we shall discuss the function $f(\alpha, s)$ rather than the weight function $f(\alpha,-s)$ of the expansion (7.1).

By multiplying both sides of Eq. (6.41) by the operator $F$ and forming the trace of the resulting relation, we find that for different values of the order parameter $s$ the functions $f(\alpha, s)$ are related by the simple Gaussian convolution

$$
\begin{array}{r}
f(\alpha, s)=\frac{2}{t-s} \int f\left(\alpha^{\prime}, t\right) \exp \left(-\frac{2\left|\alpha-\alpha^{\prime}\right|^{2}}{t-s}\right) \frac{d^{2} \alpha^{\prime}}{\pi} \\
\text { for } \operatorname{Res}<\operatorname{Ret} .
\end{array}
$$

This relation makes it clear that if the function $f(\alpha, t)$ is well behaved, then so is the function $f(\alpha, s)$ for Res $\leq$ Ret.

By performing a similar operation upon Eq. (6.42) we find that the function $f(\alpha, s)$ satisfies the differential equation

$$
\frac{\partial f(\alpha, s)}{\partial s}=-\frac{1}{2} \frac{\partial^{2} f(\alpha, s)}{\partial \alpha \partial \alpha^{*}}
$$

which is the differential form of Eq. (7.32). The relations (7.33) and (7.32) have the same form as the heatdiffusion equation and its solution. This analogy becomes more complete when the operator $F$ is a density operator; we shall discuss it in that context in the paper which follows.

From Eq. (6.46) we find that the function $f(\alpha, s)$ is normalized in the sense that

$$
\operatorname{Tr} F=\int f(\alpha, s) \pi^{-1} d^{2} \alpha
$$

subject to the existence of the trace and the convergence of the integral. This expression for the trace of an operator is a generalization of the earlier one (2.28) which may be recovered by putting $s=-1$.

Let us now use the properties of the operator $T(\alpha, s)$ which were discussed in Sec. VI to characterize the behavior of the function $f(\alpha, s)=\operatorname{Tr}[F T(\alpha, s)]$ as a function of the order parameter $s$. We first focus our attention primarily upon the left half-plane Res $<0$ where, as we shall show, the function $f(\alpha, s)$ is a bounded, square-integrable, and infinitely differentiable function of $\alpha$ for all bounded operators $F$. We shall then examine the changes in the properties of the function $f(\alpha, s)$ as the real part of $s$ becomes positive.

We have noted earlier that the operator $T(\alpha, s)$ is bounded for $\operatorname{Re} s<0$, as is shown by the estimate (6.30) for its Hilbert-Schmidt norm $\|T(\alpha, s)\|$. It follows, therefore, from the inequality (3.24) that if the operator $F$ is bounded with norm $\|F\|$, then the modulus of the function $f(\alpha, s)$ is bounded for Res $<0$ by the quantity

$$
\begin{aligned}
|f(\alpha, s)|=|\operatorname{Tr}[F T(\alpha, s)]| \leq\|F\|\|T(\alpha, s)\| \\
\leq\|F\| /(-\mathrm{Res})^{1 / 2},
\end{aligned}
$$

which is independent of $\alpha$.

Let us now observe that by using the identity (3.6) and the definition (6.3) we may obtain the relation

$$
\begin{aligned}
\int|f(\alpha, s)|^{2} d^{2} \alpha & =\int|\operatorname{Tr}[F D(\xi, s)]|^{2} d^{2} \xi \\
& =\int e^{\operatorname{Re} s|\xi|^{2}}|\operatorname{Tr}[F D(\xi)]|^{2} d^{2} \xi
\end{aligned}
$$

which for Res $\leq 0$ implies the inequality

$$
\int|f(\alpha, s)|^{2} d^{2} \alpha \leq \int|\operatorname{Tr}[F D(\xi)]|^{2} d^{2} \xi .
$$

When the operator $F$ is bounded, the integral on the right-hand side of this equation converges, as is shown by Eq. (3.16). Thus for all bounded operators $F$, the function $f(\alpha, s)$ is a square-integrable function of $\alpha$ for Res $\leq 0$. By comparing Eq. (7.37) with Eq. (3.16), we find for the norm $\|f(\alpha, s)\|$ of the function $f(\alpha, s)$, defined by Eq. (3.8), the inequality

$$
\|f(\alpha, s)\| \leq\|F\| \text { for } \operatorname{Re} s \leq 0 .
$$

We may infer from the relation (7.36) that the regions in the $s$ plane in which the functions $f(\alpha, s)$ and $\operatorname{Tr}[F D(\xi, s)]$ are square-integrable are identical and are bounded by a straight line on which the real part of $s$ is constant. Let us denote this line by $\operatorname{Re} s=x(F)$. Then for bounded operators $F$ we have $x(F) \geq 0$ since the function $f(\alpha, s)$ is in $L_{2}$ at least for $\operatorname{Res} \leq 0$.

By differentiating both sides of the relation (6.3), we may express the derivatives of the function $f(\alpha, s)$ as the 
integrals

$$
\begin{aligned}
\frac{\partial^{l+n+m} f(\alpha, s)}{\partial s^{l} \partial \alpha^{n} \partial \alpha^{* m}}=\int\left(\frac{1}{2}|\xi|^{2}\right)^{l}\left(\xi^{*}\right)^{n}(-\xi)^{m} \\
\\
\times \operatorname{Tr}[F D(\xi, s)] e^{\alpha \xi^{*-\alpha^{*}} d^{2} \xi / \pi .}
\end{aligned}
$$

The moduli of these derivatives are, accordingly, bounded by the integrals

$$
\begin{aligned}
\left|\frac{\partial^{l+n+m} f(\alpha, s)}{\partial s^{l} \partial \alpha^{n} \partial \alpha^{* m}}\right| \leq\left(\frac{1}{2}\right)^{l} \int|\xi|^{2 l+n+m} e^{\operatorname{Re} s|\xi|^{2}} \\
\times|\operatorname{Tr}[F D(\xi)]| d^{2} \xi / \pi
\end{aligned}
$$

which are independent of $\alpha$. According to the Schwarz inequality for functions (3.9), the convergence of these integrals for $\operatorname{Res}<0$ and for all bounded operators $F$ is insured by the exponential factor $\exp \left(\operatorname{Res}|\xi|^{2}\right)$ and the square-integrability of the function $\operatorname{Tr}[F D(\xi)]$. A similar argument ${ }^{2}$ shows that the derivatives (7.39) exist and are bounded for $\operatorname{Re} s<x(F)$.

We may conclude, therefore, that for $\operatorname{Re}<x(F)$, a region which includes the half-plane $\operatorname{Res}<0$ if $F$ is bounded, the function $f(\alpha, s)$ possesses derivatives of all orders with respect to $s, \alpha$, and $\alpha^{*}$ and that these derivatives are bounded by quantities that are independent of $\alpha$. In particular the function $f(\alpha, s)$ is an analytic function of $s$ for $\operatorname{Re} s<x(F)$ and its modulus is bounded by a quantity $M(s)$ which depends upon $s$ but not upon $\alpha$; i.e., we have

$$
|f(\alpha, s)| \leq M(s) \text { for } \operatorname{Res}<x(F) .
$$

It may be shown ${ }^{2}$ that the Taylor series in $s, \alpha$, and $\alpha^{*}$ formed with the derivatives (7.39) converges for all $\alpha$ and all $s$ such that $\operatorname{Re} s<x(F)$.

We recall that the existence of derivatives of all orders with respect to $\alpha$ and $\alpha^{*}$ is required if the function $f(\alpha,-s)$ is to be a generating function for the coefficients $f_{n, m}(s)$ according to the rule (7.10). We also note that the convergence of the power series for $f(\alpha,-s)$ guarantees that the function $f(\alpha,-s)$ is well defined by the correspondence $C(s)$ in the form of Eqs. (7.5)-(7.7). Finally, since for $F$ bounded and $\operatorname{Res}<0$ the derivatives of $f(\alpha,-s)$ with respect to $\alpha$ and $\alpha^{*}$ are analytic functions of $s$, our earlier observation that for $F$ bounded and Res $>0$ the coefficients $f_{n, m}(s)$ are analytic is confirmed.

As the parameter $s$ crosses the line $\operatorname{Re} s=0$, the class of operators $F$ for which the function $f(\alpha, s)$ $=\operatorname{Tr}[F T(\alpha, s)]$ is bounded shrinks dramatically. We have seen in Eq. (7.35) that this function is bounded for all bounded operators when $\operatorname{Re} s<0$. Let us now note that the function $f(\alpha, s)$ is bounded even for all finite operators for $\operatorname{Re}<<0$. According to Eq. (6.32) the operator $T(\alpha, s)$ is in the trace class for Res $<0$. It follows, therefore, from the inequality (3.39) that if the operator $F$ is finite with norm $\|F\|_{1}$, as defined by Eq. (3.33), then the modulus of the function $f(\alpha, s)$ is bounded for Res $<0$ by the quantity

$$
\begin{array}{r}
|f(\alpha, s)|=|\operatorname{Tr}[F T(\alpha, s)]| \leq\|F\|_{1}\|T(\alpha, s)\|_{2} \\
\leq 2\|F\|_{1} /(|1-s|-|1+s|),
\end{array}
$$

which is independent of $\alpha$.

On the line $\operatorname{Re}=0$, however, the operator $T(\alpha, s)$ is finite but not bounded and the function $f(\alpha, s)$ is not necessarily bounded for finite operators or even for bounded operators. It is in general necessary for the operator $F$ to be a trace-class operator if the function $f(\alpha, s)$ is to be bounded for Res $=0$. In this case, by using the inequality (3.39) and the estimate (6.31) for the norm $\|T(\alpha, s)\|_{1}$, defined in Eq. (3.33), we may obtain for the function $f(\alpha, s)$ the upper bound

$$
\begin{array}{r}
|f(\alpha, s)|=|\operatorname{Tr}[F T(\alpha, s)]| \leq\|F\|_{2}\|T(\alpha, s)\|_{1} \\
\leq 2\|F\|_{2} /|1-s| \text { for } \operatorname{Res} \leq 0,
\end{array}
$$

where $\|F\|_{2}$ is the trace-class norm of the operator $F$, a norm which we defined in Eq. (3.37). For Res $>0$ the function $f(\alpha, s)$ is not in general bounded even for traceclass operators.

We have seen that for all bounded operators $F$ the function $f(\alpha, s)$ possesses derivatives of all orders with respect to the variables $s, \alpha$, and $\alpha^{*}$ for $\operatorname{Re} s<0$. For Res $=0$, however, the function $f(\alpha, s)$ is not even a continuous function of $\alpha$ for all bounded operators. It is in general necessary for the operator $F$ to be a traceclass operator for the function $f(\alpha, s)$ to be continuous on the line $\operatorname{Re} s=0$, where it may be shown ${ }^{2}$ to be uniformly continuous.

For Res $>0$ the operator $T(\alpha, s)$ is not a finite operator and the three norms $\|T(\alpha, s)\|,\|T(\alpha, s)\|_{1},\|T(\alpha, s)\|_{2}$, which are defined by Eqs. (3.15), (3.33), and (3.37), are all infinite. At $s=1$ all of the eigenvalues $e_{n}(s)$ of the operator $T(\alpha, s)$ are infinite as may be seen from Eq. (6.28). For these reasons the function

$$
f(\alpha, s)=\operatorname{Tr}[F T(\alpha, s)]
$$

typically develops singularities of some type at one or more points in the half-plane Res $>0$. Since the function $f(\alpha, s)$ is bounded, square-integrable, and infinitely differentiable for $\operatorname{Re} s<x(F)$, this singularity must occur at a value of $s=s_{0}$ for which $\operatorname{Res}_{0} \geq x(F)$. The useful properties that we have attributed to the function $f(\alpha, s)$ cease to hold if not at the line $\operatorname{Re} s=x(F)$ then certainly at the appearance of the first singularity. For larger values of Res the function $f(\alpha, s)$ is simply too singular to be used either as a weight function for the operator $F$ in the expansion (7.1) or as a generating function for the coefficients of its $s$-ordered power-series expansion. A further discussion of this problem in terms of distribution theory is given in Ref. 2, where it is shown that the function $f(\alpha, s)$ lies outside the space of 
tempered distributions ${ }^{26}$ for $\operatorname{Re} s>\operatorname{Re} s_{0}$ where $s_{0}$ is the first singular point.

The locations of the line Res $=x(F)$ and of the singularities of the function $f(\alpha, s)$ vary considerably from one operator to another. As our example in Sec. VIII will show, the quantities $\operatorname{Re}_{0}$ and $x(F)$ may be arbitrarily small even when the operator $F$ is in the trace class.

\section{ILLUSTRATION OF OPERATOR- FUNCTION CORRESPONDENCES}

We shall now illustrate the results of Secs. VI and VII on the representation of operators by considering a simple example in some detail. This example will show how the weight functions $f(\alpha, s)=\operatorname{Tr}[F T(\alpha, s)]$, which are extremely well behaved for $\operatorname{Res}<0$, can develop singularities for $\operatorname{Res}>0$ even when the operator $F$ is both bounded and in the trace class. As we mentioned earlier, these singularities reflect the fact that $T(\alpha, s)$ is a finite operator only for $\operatorname{Re} s \leq 0$.

Let us consider again the example provided by the operator $F(\lambda)=\lambda^{a \dagger a}$. Since the operator $F(\lambda)$ may be written, according to Eq. (6.22), as

with

$$
F(\lambda)=\frac{1}{2}(1-t) T(0, t),
$$

$$
t=(\lambda+1) /(\lambda-1),
$$

we may write the function

$$
f_{\lambda}(\alpha, s)=\operatorname{Tr}[F(\lambda) T(\alpha, s)]
$$

in the form

$$
f_{\lambda}(\alpha, s)=\frac{1}{2}(1-t) \operatorname{Tr}[T(0, t) T(\alpha, s)] .
$$

The trace may be evaluated by means of Eq. (6.39), which yields

$$
\begin{aligned}
f_{\lambda}(\alpha, s) & =\frac{t-1}{t+s} \exp \left(\frac{2|\alpha|^{2}}{t+s}\right) \\
& =\frac{2}{1+\lambda-s+s \lambda} \exp \left(\frac{2(\lambda-1)|\alpha|^{2}}{1+\lambda-s+s \lambda}\right) .
\end{aligned}
$$

The function $f_{\lambda}(\alpha, s)$ is an analytic function of $s$ except for an essential singularity at

$$
s=s_{0}=(1+\lambda) /(1-\lambda) \text {. }
$$

As a function of $\alpha$, it is for $\operatorname{Re}<<\operatorname{Res}_{0}$ a Gaussian function with its maximum at $\alpha=0$. The function $f_{\lambda}(\alpha, s)$ is accordingly a bounded, square-integrable, and infinitely differentiable function of $\alpha$ for $\operatorname{Res}<\operatorname{Res}_{0}$. Thus, for the operator $F(\lambda)$ the quantity $x[F(\lambda)]$, introduced

${ }^{26}$ Tempered distributions form a class of continuous linear functionals which includes the $\delta$ function and its derivatives. A distribution is said to be tempered if it can be expressed as a derivative of finite order of a continuous function that is bounded by a polynomial. See, e.g., I. M. Gel'Fand and G. E. Shilov, Generalized Functions, translated by E. Saletan (Academic Press Inc., New York, 1964), Vol. I. in Sec. VII, is given by

$$
\begin{aligned}
x[F(\lambda)] & =\operatorname{Res}_{0}=\operatorname{Re}\left(\frac{1+\lambda}{1-\lambda}\right) \\
& =\left(1-|\lambda|^{2}\right) /|1-\lambda|^{2} .
\end{aligned}
$$

Let us recall that the operator $F(\lambda)$ is both bounded and in the trace class for $|\lambda|<1$, that it is finite but not bounded for $|\lambda|=1$, and that it is neither finite nor bounded for $|\lambda|>1$. From Eq. (8.7) we see that the parameter $x$ is positive for $|\lambda|<1$, zero for $|\lambda|=1$, and negative for $|\lambda|>1$. The region $\operatorname{Res}<x$, in which the function $f_{\lambda}(\alpha, s)$ is well behaved, therefore includes the half-plane Res $\leq 0$ when and only when the operator $F(\lambda)$ is both bounded and in the trace class. Under the transformation (8.6), the location $s_{0}$ of the singularity in the function $f_{\lambda}(\alpha, s)$ assumes every value in the halfplane $\operatorname{Res}>0$ as the parameter $\lambda$ ranges over the disk $|\lambda|<1$. Thus there are values of $\lambda$ for which the operator $F(\lambda)$ is in the trace class but for which the line $\operatorname{Re} s=x$, on which the singular point $s_{0}$ falls, lies arbitrarily close to the imaginary axis $\operatorname{Re} s=0$.

According to Eq. (6.11) the operator $F(\lambda)$ may be expanded in the form

$$
\begin{aligned}
F(\lambda) & =\lambda^{a^{\dagger a}} \\
& =\int f_{\lambda}(\alpha,-s) T(\alpha, s) \pi^{-1} d^{2} \alpha
\end{aligned}
$$

where the weight function $f_{\lambda}(\alpha,-s)$, which is given by Eq. (8.5), is the one associated with the operator $F(\lambda)$ by the correspondence $C(s)$. By comparing Eq. (8.5), after substituting $-s$ for $s$, with the $s$-ordered powerseries expansion for the operator $F(\lambda)$, Eq. (5.27), we may verify that the association of the weight function $f_{\lambda}(\alpha,-s)$ with the operator $F(\lambda)$ is in accordance with the rules (7.5) and (7.6) of the correspondence $C(s)$.

We see from Eq. (8.5) that for $\operatorname{Re} s>\operatorname{Re}_{0}$ the function $f_{\lambda}(\alpha, s)$ increases for large values of $|\alpha|$ as an exponential function of $|\alpha|^{2}$. For this reason the function $f_{\lambda}(\alpha, s)$ is a tempered distribution only for $\operatorname{Re} \leq \leq \operatorname{Res}_{0}{ }^{26}$ Now we have seen that as the parameter $\lambda$ ranges over the disk $|\lambda|<1$ in which the operator $F(\lambda)$ is both bounded and in the trace class, the singularity at $s_{0}=(1+\lambda)(1-\lambda)^{-1}$ ranges over the half-plane $\operatorname{Re} s>0$. From this counterexample we may conclude that for no value of $\operatorname{Res}<0$ do the operators $T(\alpha, s)$ afford a basis for the integral representation (6.11) of an arbitrary bounded operator $F$ even if the whole class of tempered distributions is admitted as weight functions. Thus, the operators $T(\alpha, s)$ for $\operatorname{Res}<0$ contrast sharply with the displacement operators $D(\alpha)$ in terms of which every bounded operator may be expanded with a square-integrable weight function. In this sense the operators $T(\alpha, s)$ for $\operatorname{Res}<0$ must be regarded as undercomplete. 
We may mention that it is not difficult to show ${ }^{2}$ that the weight function $f(\alpha,-s)$ may, for all values of $s$ and all bounded operators $F$, be interpreted as a member of the space ${ }^{27}$ of ultradistributions $Z^{\prime}$. In order to accommodate such weight functions, however, the structure of the representation (6.11) must be changed in a major way. When the operator $F$ is in the trace class, the additional terms required to regularize the representation (6.11) may be found by generalizing a procedure formulated ${ }^{28}$ for the case of the $P$ representation of the density operator, Eqs. (7.21) and (7.22).

\section{APPENDIX A}

Our object here is to derive the useful integral identity $^{7}$

$$
\begin{array}{r}
z^{-1} f\left(z^{-1} y\right)=\int f(\alpha) \exp \left(\alpha^{*} y-z|\alpha|^{2}\right) \pi^{-1} d^{2} \alpha, \\
\operatorname{Re} z>0,
\end{array}
$$

which holds for all entire functions $f(\alpha)$ subject to appropriate conditions on the convergence of the integral. We evaluate first the integral $K_{n}$ :

$$
\begin{aligned}
K_{n} & =\int \alpha^{n} \exp \left(\alpha^{*} y-z|\alpha|^{2}\right) \pi^{-1} d^{2} \alpha \\
& =(n !)^{-1} y^{n} \int|\alpha|^{2 n} e^{-z|\alpha|^{2}} \pi^{-1} d^{2} \alpha \\
& =z^{-1}\left(z^{-1} y\right)^{n} .
\end{aligned}
$$

Then by writing

$$
f(\alpha)=\sum_{n=0}^{\infty} c_{n} \alpha^{n}
$$

we find that

$$
\begin{aligned}
\int e^{\alpha^{*} y-z|\alpha|^{2}} f(\alpha) \pi^{-1} d^{2} \alpha & =\sum_{n=0}^{\infty} c_{n} K_{n} \\
& =z^{-1} f\left(z^{-1} y\right)
\end{aligned}
$$

which is Eq. (A1), provided that the integral may be done term by term. In particular, if we let $f(\alpha)=\exp (\alpha x)$, then Eq. (A1) becomes

$$
z^{-1} \exp \left(z^{-1} x y\right)=\int \exp \left(\alpha x+\alpha^{*} y-z|\alpha|^{2}\right) \pi^{-1} d^{2} \alpha,
$$

provided $\operatorname{Re} z>0$.

\footnotetext{
${ }^{27}$ The space $Z^{\prime}$ of ultradistributions is a class of generalized functions which includes but is much larger than the space of tempered distributions. It is discussed in the work by Gel'Fand and Shilov (Ref. 26).

${ }_{28} \mathrm{~K}$. E. Cahill (to be published).
}

\section{APPENDIX B}

We shall now express the matrix elements of the displacement operator $D(\alpha)$ in the $n$-quantum representation in terms of the associated Laguerre polynomials $L_{n}{ }^{(m)}(x)$. We first note that if $|\alpha\rangle$ is a coherent state then, by using Eqs. (2.19) and (2.20), we may write

$$
\begin{aligned}
D(\xi)|\alpha\rangle & =D(\xi) D(\alpha)|0\rangle \\
& =D(\xi+\alpha)|0\rangle \exp \left[\frac{1}{2}\left(\xi \alpha^{*}-\xi^{*} \alpha\right)\right] \\
& =|\xi+\alpha\rangle \exp \left[\frac{1}{2}\left(\xi \alpha^{*}-\xi^{*} \alpha\right)\right] .
\end{aligned}
$$

Thus, by using Eq. (2.23), we find that

$$
\begin{aligned}
&\langle m|D(\xi)| \alpha\rangle=(m !)^{-1 / 2}(\xi+\alpha)^{m} \exp \left[\frac{1}{2}\left(\xi \alpha^{*}-\xi^{*} \alpha\right)\right. \\
&\left.-\frac{1}{2}|\xi+\alpha|^{2}\right] \\
&=(m !)^{-1 / 2}(\xi+\alpha)^{m} \exp \left(-\frac{1}{2}|\xi|^{2}-\frac{1}{2}|\alpha|^{2}\right. \\
&\left.-\xi^{*} \alpha\right) .
\end{aligned}
$$

Another expression for this same matrix element also follows from Eq. (2.23):

$$
\langle m|D(\xi)| \alpha\rangle=e^{-|\alpha|^{2} / 2} \sum_{m=0}^{\infty} \frac{\alpha^{n}}{(n !)^{1 / 2}}\langle m|D(\xi)| n\rangle .
$$

If we now combine Eqs. (B1) and (B2) and put $y=\xi^{-1} \alpha$, we arrive at the relation

$$
\begin{aligned}
(1+y)^{m} e^{-y|\xi|^{2}}=e^{|\xi|^{2} / 2} \sum_{n=0}^{\infty}\left(\frac{m !}{n !}\right)^{1 / 2} \xi^{n-m} & \\
& \times\langle m|D(\xi)| n\rangle y^{n} .
\end{aligned}
$$

The left-hand side of this equation is a generating function for the associated Laguerre polynomials $L_{n}{ }^{(m)}(x)$ according to the identity ${ }^{19}$

$$
(1+y)^{m} e^{-x y}=\sum_{n=0}^{\infty} L_{n}^{(m-n)}(x) y^{n},
$$

which holds for $|y|<1$. Thus by comparing Eqs. (B3) and (B4) we obtain the expression

$$
\langle m|D(\xi)| n\rangle=(n ! / m !)^{1 / 2} \xi^{m-n} e^{-|\xi|^{2} / 2} L_{n}{ }^{(m-n)}\left(|\xi|^{2}\right) .
$$

\section{APPENDIX C}

Our definition (5.6) of the $s$-ordered product was as the derivative

$$
\left\{\left(a^{\dagger}\right)^{n} a^{m}\right\}_{s}=\left.\frac{\partial^{n+m} D(\alpha, s)}{\partial \alpha^{n} \partial\left(-\alpha^{*}\right)^{m}}\right|_{\alpha=0} .
$$

By using Eq. (5.1) we may write the operator $D(\alpha, s)$ as a $t$-ordered exponential

$$
\begin{aligned}
D(\alpha, s) & =D(\alpha, t) e^{(s-t)|\alpha|^{2} / 2} \\
& =\left\{\exp \left[\alpha a^{\dagger}-\alpha^{*} a+\frac{1}{2}(s-t)|\alpha|^{2}\right]\right\}_{t} .
\end{aligned}
$$


We note the following equivalent expansions:

$$
\begin{aligned}
e^{\lambda w+\mu z+\nu w z} & =\sum_{m=0}^{\infty} \frac{z^{m}}{m !}(\mu+\nu w)^{m} e^{\lambda w} \\
& =\sum_{n=0}^{\infty} \frac{w^{n}}{n !}(\lambda+\nu z)^{n} e^{\mu z} .
\end{aligned}
$$

By using the identity (B4), we may write these expansion in the forms

$$
\begin{aligned}
e^{\lambda w+\mu z+\nu w z} & =\sum_{n, m=0}^{\infty} \frac{z^{m}}{m !} w^{n} \mu^{m-n} \nu^{n} L_{n}{ }^{(m-n)}(-\lambda \mu / \nu) \\
& =\sum_{n, m=0}^{\infty} \frac{w^{n}}{n !} z^{m} \lambda^{n-m} \nu^{m} L_{m}{ }^{(n-m)}(-\lambda \mu / \nu) .
\end{aligned}
$$

Let us make the identifications

$$
\begin{aligned}
w=\alpha, & \lambda=a^{\dagger}, \quad \nu=-\frac{1}{2}(s-t), \\
z=\alpha^{*}, & \mu=a,
\end{aligned}
$$

in Eqs. (C4a) and (C4b) and specify $t$ ordering of both sides of these equations. Then according to Eq. (C2) we have found the $t$-ordered form for the displacement operator $D(\alpha, s)$. By carrying out the differentiations indicated in Eq. (C1) we find from the expansion (C4a) the result

$$
\left\{\left(a^{\dagger}\right)^{n} a^{m}\right\}_{s}=n !\left(\frac{t-s}{2}\right)^{n}\left\{a^{m-n} L_{n}{ }^{(m-n)}\left(\frac{2 a^{\dagger} a}{s-t}\right)\right\}_{t}
$$

which is useful for $m \geq n$, and from (C4b)

$$
\left\{\left(a^{\dagger}\right)^{n} a^{m}\right\}_{s}=m !\left(\frac{t-s}{2}\right)^{m}\left\{\left(a^{\dagger}\right)^{n-m} L_{m}{ }^{(n-m)}\left(\frac{2 a^{\dagger} a}{s-t}\right)\right\}_{t}
$$

which is useful for $n \geq m$. These two expressions corre- spond to the explicit expansion

$$
\begin{aligned}
\left\{\left(a^{\dagger}\right)^{n} a^{m}\right\}_{8}= & \sum_{k=0}^{(n, m)} k !\left(\begin{array}{l}
n \\
k
\end{array}\right)\left(\begin{array}{l}
m \\
k
\end{array}\right)\left(\frac{t-s}{2}\right)^{k} \\
& \times\left\{\left(a^{\dagger}\right)^{n-k} a^{m-k}\right\}_{t},
\end{aligned}
$$

which is noted in Eq. (5.12).

\section{APPENDIX D}

By expanding the coherent states $|\beta\rangle$ and $\langle\beta|$ of Eq. (6.36) in terms of the $n$-quantum states, we find

$$
\begin{aligned}
e^{|\beta|^{2}}\langle\beta|T(\alpha, s)| \beta\rangle & =\sum_{n, m=0}^{\infty}(n ! m !)^{-1 / 2}\left(\beta^{*}\right)^{n} \beta^{m}\langle n|T(\alpha, s)| m\rangle \\
& =\frac{2}{1-s} \exp \left(|\beta|^{2}-\frac{2|\alpha-\beta|^{2}}{1-s}\right)
\end{aligned}
$$

If we now make use of the expansion ( $\mathrm{C} 4 \mathrm{a}$ ) with the identifications

$$
\begin{aligned}
w=\beta^{*}, & \lambda=2 \alpha /(1-s), \quad \nu=-(1+s) /(1-s), \\
z=\beta, & \mu=2 \alpha^{*} /(1-s),
\end{aligned}
$$

then we find

$$
\begin{aligned}
e^{|\beta|^{2}}\langle\beta|T(\alpha, s)| \beta\rangle= & \frac{2}{1-s} \exp \left(-\frac{2|\alpha|^{2}}{1-s}\right) \sum_{n, m=0}^{\infty} \frac{\left(-\beta^{*}\right)^{n} \beta^{m}}{m !} \\
& \times\left(\frac{2 \alpha^{*}}{1-s}\right)^{m-n}\left(\frac{1+s}{1-s}\right)^{n} L_{n}{ }^{(m-n)}\left(\frac{4|\alpha|^{2}}{1-s^{2}}\right) .
\end{aligned}
$$

Hence we have

$$
\begin{aligned}
&\langle n|T(\alpha, s)| m\rangle=\frac{n !}{m !}\left(\frac{2}{1-s}\right)^{m-n+1}\left(\frac{s+1}{s-1}\right)^{n}\left(\alpha^{*}\right)^{m-n} \\
& \quad \times \exp \left(-\frac{2|\alpha|^{2}}{1-s}\right) L_{n}{ }^{(m-n)}\left(\frac{4|\alpha|^{2}}{1-s^{2}}\right),
\end{aligned}
$$

which is Eq. (6.51). 